\title{
杂环酮腙与类似物的合成及其对结核杆菌的抑制活性
}

\author{
李忠洲 ${ }^{a}$ 赵 升 ${ }^{a}$ 李新军 ${ }^{a}$ 王翠翠 ${ }^{a}$ \\ 姚璐璐 $a$ 金瑞良 $b$ 邹新琢 $*, a$ \\ ( ${ }^{a}$ 华东师范大学化学系 上海 200241) \\ ( ${ }^{b}$ 同济大学附属上海肺科医院上海市结核病(肺)重点实验室 上海 200433)
}

\begin{abstract}
摘要 22 个新的杂环酮腙及其类似物被合成, 其对结核分枝杆菌菌株 $H 37 R v$ 的抑菌活性被测定. 这些化合物都显示出 抑菌活性. 其中, 化合物( $1 E, 4 E$ )-1,5-二(5'-澳-2'-噻吩基)-1,4-戊二烯-3-酮-(1',4',5',6'-四氢嘧啶基)腙(1d)的 $\mathrm{MIC}_{90}$ 为 $2 \mu \mathrm{g} /$ $\mathrm{mL}$, 显示出最高的抑菌活性; 同时该化合物对单耐异烟肼菌株 242、耐多药菌株 2312 和广泛耐药菌株 1220 的 $\mathrm{MIC}_{90}$ 值分别为 $0.25,1.0$ 和 $0.5 \mu \mathrm{g} / \mathrm{mL}$, 也表现出很好的抑菌作用.
\end{abstract}

关键词 杂环酮腙; 合成; 结核分枝杆菌; 抑菌活性

\section{Synthesis and Anti-mycobacterial Activities of Heterocyclic Hydrazones and Their Bioisosteric Compounds}

\author{
Li, Zhongzhou $^{a} \quad$ Zhao, Sheng $^{a} \quad$ Li, Xinjun $^{a} \quad$ Wang, Cuicui ${ }^{a}$ \\ Yao, Lulu $^{a} \quad$ Jin, Ruiliang ${ }^{b} \quad$ Zou, Xinzhuo ${ }^{*, a}$ \\ ( ${ }^{a}$ Department of Chemistry, East China Normal University, Shanghai 200241) \\ $\left({ }^{b}\right.$ Shanghai Key Laboratory of Tuberculosis, Shanghai Pulmonary Hospital, Tongji University, Shanghai 200433)
}

\begin{abstract}
A total of 22 new heterocyclic hydrazones and their bioisosteric compounds were synthesized and evaluated for their activity against Mycobacterium tuberculosis, strain H37Rv. All newly synthesized compounds showed inhibitory activities. Among these compounds, (1E,4E)-1,5-bis(5-bromo-2-thiophenyl)-1,4-pentadiene-3-one-(1,4,5,6-tetrahydro-2-pyrimidimyl)-hydrazone (1d) showed the most effective anti-tubercular activity with $\mathrm{MIC}_{90}$ value of $2 \mu \mathrm{g} / \mathrm{mL}$, and exhibited an excellent inhibitory activity against isoniazid-resistant strain 242 , multidrug-resistant strain 2312 , and extensively drug-resistant strain 1220 with $\mathrm{MIC}_{90}$ values of $0.25,1.0$ and $0.5 \mu \mathrm{g} / \mathrm{mL}$, respectively.
\end{abstract}

Keywords heterocyclic hydrazone analogs; synthesis; Mycobacterium tuberculosis; anti-mycobacterial activity

肺结核(TB)是由结核分枝杆菌(MTB)引起的一种传 染病, 被称为传染病中的头号杀手, 曾给人类带来了巨 大的灾难. 利福平、异烟肼、乙胺丁醇等特效药物的成 功开发及其有效的组合疗法 ${ }^{[1,2]}$, 使该病得到有效控制. 近年来，由于这些药物的长期的使用，产生了单耐、耐 多药和死亡率极高的广泛等耐药菌 ${ }^{[3 \sim 6]}$; 此外, 艾滋病 感染并发症的传播 ${ }^{[7}$ 9]等因素, 使防治的难度增大. 2012 年, 全球有将近 860 万人受到感染, 约 130 万人死 于此 病 $^{[10 \sim 13]}$. 此外, 现有的结核病治疗方法疗程长, 至少需要 6 个月以上, 易引起眼、肝炎等产生药物不良 反应 ${ }^{[1,14,15]}$. 结核病防治形势严峻性近年来受到关注, 抗结核病新药的研发速度明显加快, 已有多种候选药物

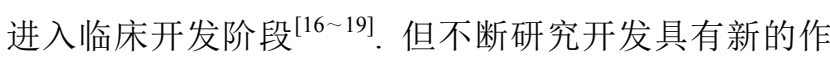
用机制、药效好且对耐多药菌和广泛耐药菌株也同样有 效、安全、低成本的新药物仍是目前的重要目标 ${ }^{[20,21]}$. 腙 类化合物由于具有抗菌、抗 HIV、抗病毒以及抗肿瘤等 多种广泛生理活性, 近年来其研究受到重视 ${ }^{[22]}$; 一些腙 类化合物还具有杀虫活性 ${ }^{[23]}$. 赵升等为了寻找有灭杀 白蚁活性的化合物, 合成了一些取代芳基烯酮腙类化合 物 ${ }^{[24]}$; 而异烟腙是一个早期治疗肺结核的药物 ${ }^{[25]}$. 因为 二者化学结构较为相似, 我们推测杂环酮腙类化合物 也可能对结核菌也具有抑菌活性, 于是设计和合成了 一些相关的杂环酮腙及其类似化合物(图 1), 并测定 了这些化合物对结核分枝杆菌菌株 H37Rv (ATCC

\footnotetext{
*E-mail:xzzou@chem.ecnu.edu.cn

Received December 4, 2014; revised January 21, 2015; published online February 2, 2015.
} 
27294)的抑菌活性(表 1).<smiles>COc1cc(/C=N/NC(=O)c2ccncc2)ccc1O</smiles><smiles>[X]C=C(NN)N[In]</smiles><smiles>[Z]C=CC([Y])(O)C[Z]</smiles>

$2 a \sim 2 e$

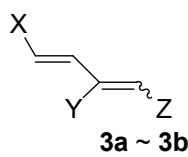

图 1 异烟腙和合成化合物的化学结构式

Figure 1 Chemical structures of isoniazone and synthesized compounds

\section{1 结果与讨论}

首先, 我们设计了一些由芳香杂环、氢化嘧啶和氢 化咪唑等脂环类杂环构成的酮腙化合物 $\mathbf{1 a} \sim \mathbf{1 p}$ (图 1 和 表 1). 为了比较, 也合成了一个含苯环的酮腙化合物 $\mathbf{1 i}$. 这些化合物的合成路线如 Scheme 1 所示. 结构对称的 酮腙 1a 1i 分别以相应的杂环芳香醛 4 和丙酮在 $10 \%$ 氢氧化钠溶液作用下, 以良好的收率得到结构对称的不 饱和烯酮 5. 然而, 含吡啶环的不饱和烯酮 5 未能用该 方法获得. 这是因氢氧化钠碱性较强, 导致吡啶甲醛发 生了歧化反应. 参照 Merz 等 ${ }^{[26]}$ 的方法, 选择以碱性比 较弱的三乙胺为催化剂, 吡啶甲醛和丙酮二羧酸反应, 可以中等收率得到了此烯酮. 杂环芳香醛 4 和杂环芳香 基甲酮 $\mathrm{MeCOY}$ 在氢氧化钠作用下, 以良好的收率地得 到结构不对称的烯酮 5. 进一步, 不饱和烯酮 $\mathbf{5}$ 和相应 的溴化氢肼盐 $\left(\mathrm{NH}_{2} \mathrm{NH}-\mathrm{Z} \cdot \mathrm{HBr}\right)$ 在少量的氢溴酸催化下 回流反应, 用饱和 $\mathrm{Na}_{2} \mathrm{CO}_{3}$ 溶液中和后可以良好的收率 得到酮腙化合物 $1 \mathbf{a} \sim 1 \mathbf{p}$. 实验中使用的溴化氢肼盐 $\left(\mathrm{NH}_{2} \mathrm{NH}-\mathrm{Z} \cdot \mathrm{HBr}\right)$ 按文献[27～34]方法制备得到.

实验发现一些结构对称的酮腙化合物如 $1 \mathrm{~g}$ 在二甲 亚砜(DMSO)中溶解度较小. 为此, 试探性地设计和合 成了一些具有类似杂环结构的羟胺类化合物 $2 \mathrm{a} \sim 2 \mathrm{e}$ 以 及酮腙化合物 $\mathbf{1}$ 的电子等排体化合物 $\mathbf{3 a} \sim \mathbf{3 b}$, 希望能改 善这些化合物的溶解性并考察它们的生物活性. 不饱和 烯酮 5 在二氯甲烷中和冰盐浴的条件下, 用 $\left(\mathrm{CH}_{3}\right)_{2} \mathrm{~S} /$ $\left(\mathrm{CH}_{3}\right)_{2} \mathrm{SO}_{4} / t-\mathrm{BuOK}$ 处理得到了环氧化合物 $6^{[35]}$. 这些在 邻位具有双键结构的环氧化合物 $\mathbf{6}$ 在纯化过程中易发生 重排反应 ${ }^{[36]}$, 因此未经纯化处理就直接与相应的胺类 化合物作用合成得到目标化合物 $\mathbf{2 a} \sim \mathbf{2 e}$. 化合物 $\mathbf{3}$ 由相 应的羟胺类化合物用 $\mathrm{HCl}$ 脱水得到. 所有目标化合物的 结构由 NMR 等得到表征.

目标化合物对结核分枝杆菌菌株 H37Rv 的抑菌活 性按文献报道的方法进行了测定 ${ }^{[37]}$. 其体外抑菌活性

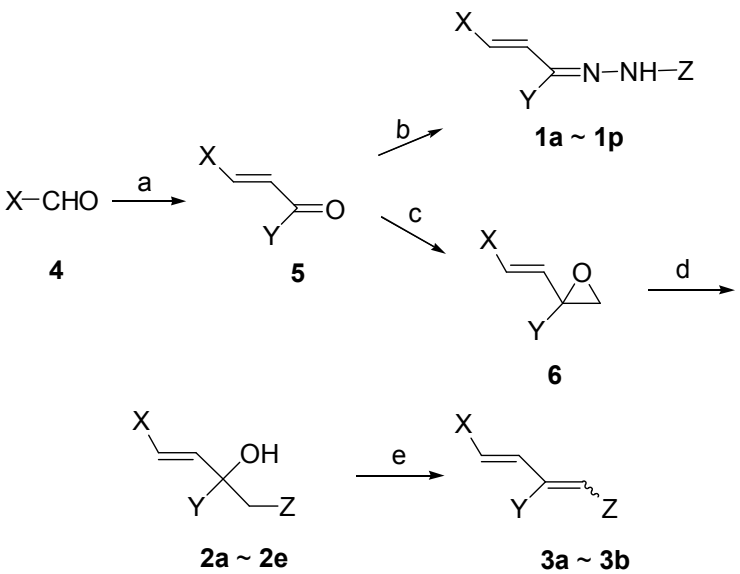

Reagents and conditions: (a) acetone or MeCOY, ethanol, 10\% $\mathrm{NaOH},-5 \sim 0{ }^{\circ} \mathrm{C}$ and then to r.t., $6 \mathrm{~h}$; (b) $\mathrm{NH}_{2} \mathrm{NH}-\mathrm{Z} \cdot \mathrm{HBr}$, ethanol, concentrated $\mathrm{HBr}$, saturated $\mathrm{Na}_{2} \mathrm{CO}_{3}$ and reflux; (c) $\left(\mathrm{CH}_{3}\right)_{2} \mathrm{~S} /\left(\mathrm{CH}_{3}\right)_{2} \mathrm{SO}_{4} / t-\mathrm{BuOK}, \mathrm{CH}_{2} \mathrm{Cl}_{2}$, ice-salt bath; (d) amine (1,4,5,6-tetrahydropyrimidin-2-amine, pyrimidin-2-amine, pyrazin-2amine or $1 \mathrm{H}-1,2,4$-triazole), $\mathrm{NaOH}, \mathrm{DMF}, 50{ }^{\circ} \mathrm{C}$; (e) concentrated $\mathrm{HCl}(\mathrm{l})$ or $\mathrm{HCl}(\mathrm{g})$, THF, r.t.

图式 1 化合物 $1 \mathrm{a} \sim 1 \mathrm{p}$ 的合成路线

Scheme 1 Synthetic route of xcompounds $1 \mathbf{a} \sim \mathbf{1 p}$

的测定结果汇总在表 1 中. 含噻吩环的化合物 $1 \mathrm{~b}$ 表现出 较好的抑菌活性, 其 $\mathrm{MIC}_{90}$ 为 $8 \mu \mathrm{g} / \mathrm{mL}$. 当噻吩环被呋 喃环(1a)、吡啶环(1h)、苯环(1i)取代, 其抑菌活性都有 下降, $\mathrm{MIC}_{90}$ 分别为 $32 、 16$ 和 $32 \mu \mathrm{g} / \mathrm{mL}$. 由于化合物 $\mathbf{1 h}$ 在 DMSO 中的溶解度较小, 因此它的 $\mathrm{MIC}_{90}$ 值未能准确 测定. 比较含有 2-噻吩环的化合物 $1 \mathrm{~b} 、 1 \mathrm{c}$ 和 $1 \mathrm{~d}$, 可知 甲基的存在导致化合物 $\mathbf{1 c}$ 的抑菌活性下降 $\left(\mathrm{MIC}_{90}\right.$ 为 32 $\mu \mathrm{g} / \mathrm{mL})$; 而溴的引进使化合物 $\mathbf{1 d}$ 的抑菌活性提高 $\left(\mathrm{MIC}_{90}\right.$ 为 $\left.2 \mu \mathrm{g} / \mathrm{mL}\right)$. 比较含有 2 -噻吩环结构的化合物 1d、1e 和 1f, 发现它们的抑菌活性相差不大, $\mathrm{MIC}_{90}$ 分别 为 2、4 和 $8 \mu \mathrm{g} / \mathrm{mL}$, 都表现出较好的抑菌活性, 说明 $\mathrm{Z}$ 部分环的大小和取代基的存在与否对化合物的抑菌活 性影响不大. $Z$ 为 1,4,5,6-四氢- 2-嘧啶基时(1d), 显示出 最高的抑菌活性 $\left(\mathrm{MIC}_{90}\right.$ 为 $\left.2 \mu \mathrm{g} / \mathrm{mL}\right)$. 具有不对称取代 基的化合物 $\mathbf{1 j} \sim 1 \mathrm{p}$ 在 DMSO 中有很好的溶解性, 对结 核分枝杆菌表现出中等的抑菌活性 $\left(\mathrm{MIC}_{90}\right.$ 为 $4 \sim 32$ $\mu \mathrm{g} / \mathrm{mL})$. 其中化合物 10 显示出最高的抑制活性 $\left(\mathrm{MIC}_{90}\right.$ 为 $4 \mu \mathrm{g} / \mathrm{mL}$ ). 更换 1,4,5,6-四氢-2-嘧啶基为 1,4,5-三氢-2咪唑基(1n)或 1,4,5,6-四氢-5,5-二甲基-2-嘧啶基(1p)都会 导致其抑菌活性略有下降 $\left(\mathrm{MIC}_{90}\right.$ 为 $\left.8 \mu \mathrm{g} / \mathrm{mL}\right)$. 化合物 $\mathbf{1 j} \sim 1 \mathrm{~m}$ 的抑菌活性比相对应的化合物 $1 \mathrm{n} \sim 1 \mathrm{p}$ 略为低 些.

羟胺类化合物 $\mathbf{2 a} \sim 2 \mathrm{e}$ 也同样显示出中等的抑菌活 性, $\mathrm{MIC}_{90}$ 值为 $8 \sim 16 \mu \mathrm{g} / \mathrm{mL}$, 它们在 DMSO 中也有很好 的溶解性. 当 $\mathrm{Z}$ 为芳香杂环时的化合物 $\mathbf{2 b} \sim \mathbf{2 d}$ 的抑菌 活性比 $Z$ 为脂杂环时的化合物 $\mathbf{2 a}$ 低. 化合物 $\mathbf{2 b}$ 中的一 
表 1 杂环酮腙及其类似物对结核分枝杆菌菌株 H37Rv 的抑菌活性

Table 1 Anti-mycobacterial activities of heterocyclic hydrazones and their bioisosteric compounds against MTB (strain H37Rv)
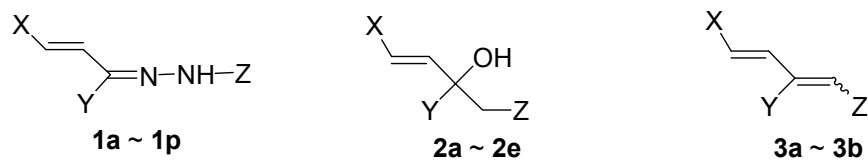

\begin{tabular}{|c|c|c|c|c|}
\hline Compd. & $\mathrm{X}$ & Y & Z & $\mathrm{MIC}_{90}{ }^{a} /\left(\mu \mathrm{g} \bullet \mathrm{mL}^{-1}\right)$ \\
\hline $1 \mathrm{a}$ & & & & 32 \\
\hline $1 b$ & & & & 8 \\
\hline 1c & & & & 32 \\
\hline 1d & & & & 2 \\
\hline $1 \mathrm{e}$ & & & & 4 \\
\hline 1f & & & & 8 \\
\hline $1 \mathrm{~g}$ & & & & $>128$ \\
\hline $1 \mathrm{~h}$ & & & & 16 \\
\hline $1 \mathrm{i}$ & & & & 32 \\
\hline $1 \mathrm{j}$ & & & & 16 \\
\hline $1 k$ & & & & 16 \\
\hline $1 \mathrm{~m}$ & & & & 32 \\
\hline 1n & & & & 8 \\
\hline 10 & & & & 4 \\
\hline $1 p$ & & & & 8 \\
\hline $2 a$ & & & & 8 \\
\hline $2 b$ & & & N- & 16 \\
\hline
\end{tabular}




Compd.

${ }^{a} \mathrm{MIC}_{90}: 90 \%$ minimal inhibitory concentration. ${ }^{b} \mathrm{SM}$ : streptomycin. ${ }^{c} \mathrm{INH}$ : isoniazid. ${ }^{d} \mathrm{RFP}$ : rifampicin. ${ }^{e}$ EMB: ethambutol.

个 4-氯苯基被 2-溴噻吩基代替时 $(2 \mathbf{e})$, 其抑制活性有所 提高. 同样，化合物 $\mathbf{3 b}$ 的活性也高于化合物 $\mathbf{3 a}$.

在所有测试的化合物中, 化合物 1d 对 MTB 显示出 最高的抑菌活性 $\left(\mathrm{MIC}_{90}\right.$ 为 $\left.2 \mu \mathrm{g} / \mathrm{mL}\right)$. 因此, 该化合物被 进一步测试了对结核分枝杆菌的耐药菌株体外活性. 结 果表明, 化合物 1d 对单耐异烟肼菌株 242 (WHO 质控菌 株)的 $\mathrm{MIC}_{90}$ 为 $0.25 \mu \mathrm{g} / \mathrm{mL}$; 耐多药菌株 2312 (上海市肺 科医院临床分离菌株)的 $\mathrm{MIC}_{90}$ 为 $1 \mu \mathrm{g} / \mathrm{mL}$; 广泛耐药菌 株 1220 (上海市肺科医院临床分离菌株)的 $\mathrm{MIC}_{90}$ 为 0.5 $\mu \mathrm{g} / \mathrm{mL}$. 即该化合物对以上耐药结核菌也同样表现出很 好的抑菌活性，其作用机制目前尚不清楚.

\section{2 结论}

22 个结构新颖的杂环酮腙及其类似物被设计和合 成, 它们对结核分枝杆菌菌株都显示出抑菌活性. 其中 化合物 1d 显示出最高的抑菌活性, 和临床药物乙胺丁 醇的活性大致相当. 令人感兴趣的是, 它对各种耐药菌 也表现出很好的抑菌作用. 该化合物结构简单, 易合成, 值得进一步研究.

\section{3 实验部分}

NMR 由 varian INOVA-500 型核磁共振仪测定, 内 标为 TMS; IR 由 NICOLET, FT-IR 红外仪测定; 元素分 析由 MOD.1106 分析仪测定; 质谱由 Agilent
HP6890-5973N 质谱仪测定; 熔点由Yanano MP 500 熔 点仪测定(未校正). 试剂为市售品, 按常规方法进行纯 化处理.

\section{1 化合物的合成}

2.1.1中间体 $(1 E, 4 E)-1,5-$ 二取代-戊二烯-3-酮 $(5 \mathbf{a} \sim$ 5e)的制备

$5 \mathrm{~mL} 10 \% \mathrm{NaOH}$ 和 $10 \mathrm{~mL}$ 乙醇的溶液, 冰盐浴冷 却至 $-5 \sim 0{ }^{\circ} \mathrm{C}$. 滴加由醛 4 (5 mmol)、丙酮 $(2.5 \mathrm{mmol})$ 和 $8 \mathrm{~mL}$ 乙醇组成的溶液. 继续反应 $6 \mathrm{~h}$ 至原料消失, 过 滤, 收集固体, 用水洗至中性, 粗产物经重结晶纯化.

$(1 E, 4 E)-1,5$-二(2'-呋喃基)-1,4-戊二烯-3-酮(5a): 收 率 $96 \%$, 黄色晶体. m.p. 49 51 ${ }^{\circ} \mathrm{C}$ (文献值 ${ }^{[33]} 50 \sim$ $\left.53{ }^{\circ} \mathrm{C}\right) ;{ }^{1} \mathrm{H}$ NMR $\left(500 \mathrm{MHz}, \mathrm{CDCl}_{3}\right) \delta: 6.48 \sim 6.49(\mathrm{~m}, 2 \mathrm{H}$, $\mathrm{FuH}), 6.67$ (d, $J=3.4 \mathrm{~Hz}, 2 \mathrm{H}, \mathrm{FuH}), 6.91$ (d, $J=15.6 \mathrm{~Hz}$, $2 \mathrm{H}, \mathrm{CH}=\mathrm{CH}), 7.47(\mathrm{~d}, J=15.6 \mathrm{~Hz}, 2 \mathrm{H}, \mathrm{CH}=\mathrm{CH}), 7.49$ (s, 2H, FuH).

$(1 E, 4 E)-1,5$-二(2'-噻吩基)-1,4-戊二烯-3-酮(5b): 收 率 92\%, 黄色晶体. m.p. $115 \sim 117{ }^{\circ} \mathrm{C}$ (文献值 ${ }^{[33]} 115 \sim$ $\left.117.5{ }^{\circ} \mathrm{C}\right) ;{ }^{1} \mathrm{H}$ NMR $\left(500 \mathrm{MHz}, \mathrm{CDCl}_{3}\right) \delta: 6.82$ (d, $J=15.6$ $\mathrm{Hz}, 2 \mathrm{H}, \mathrm{CH}=\mathrm{CH}), 7.08(\mathrm{dd}, J=3.7,5.0 \mathrm{~Hz}, 2 \mathrm{H}, \mathrm{ThH})$, $7.34(\mathrm{~d}, J=3.5 \mathrm{~Hz}, 2 \mathrm{H}, \mathrm{ThH}), 7.42(\mathrm{~d}, J=5.1 \mathrm{~Hz}, 2 \mathrm{H}$, $\mathrm{ThH}), 7.85(\mathrm{~d}, J=15.6 \mathrm{~Hz}, 2 \mathrm{H}, \mathrm{CH}=\mathrm{CH})$.

$(1 E, 4 E)-1,5$-二(5'-甲基-2'-噻吩基)-1,4-戊二烯-3-酮 $(\mathbf{5 c})^{[38]}$ : 收率 $100 \%$, 胶状物. ${ }^{1} \mathrm{H}$ NMR (500 $\left.\mathrm{MHz}, \mathrm{CDCl}_{3}\right)$ 
$\delta: 2.52\left(\mathrm{~s}, 6 \mathrm{H}, \mathrm{CH}_{3}\right), 6.67(\mathrm{~d}, J=15.6 \mathrm{~Hz}, 2 \mathrm{H}, \mathrm{CH}=\mathrm{CH})$, $6.74(\mathrm{~d}, J=2.8 \mathrm{~Hz}, 2 \mathrm{H}, \mathrm{ThH}), 7.13(\mathrm{~d}, J=3.2 \mathrm{~Hz}, 2 \mathrm{H}$, $\mathrm{ThH}), 7.75(\mathrm{~d}, J=15.6 \mathrm{~Hz}, 2 \mathrm{H}, \mathrm{CH}=\mathrm{CH})$.

(1E,4E)-1,5-二(5'-溴-2'-噻吩基)-1,4-戊二烯-3-酮 (5d $)^{[38]}$ : 收率 $82 \%$, 黄色晶体. m.p. $129 \sim 130{ }^{\circ} \mathrm{C} ;{ }^{1} \mathrm{H}$ NMR $\left(500 \mathrm{MHz}, \mathrm{CDCl}_{3}\right) \delta$ : $6.67(\mathrm{~d}, J=15.0 \mathrm{~Hz}, 2 \mathrm{H}$, $\mathrm{CH}=\mathrm{CH}), 7.04(\mathrm{~d}, J=4.0 \mathrm{~Hz}, 2 \mathrm{H}, \mathrm{ThH}), 7.08(\mathrm{~d}, J=4.0$ $\mathrm{Hz}, 2 \mathrm{H}, \mathrm{ThH}), 7.70$ (d, $J=15.0 \mathrm{~Hz}, 2 \mathrm{H}, \mathrm{CH}=\mathrm{CH})$.

(1E,4E)-1,5-二(4'-氯苯基)-1,4-戊二烯-3-酮 $(\mathbf{5 g})$ : 收 率 71\%, 黄色晶体. m.p. $193 \sim 194{ }^{\circ} \mathrm{C}$ (文献值 ${ }^{[34]} 192 \sim$ $\left.194{ }^{\circ} \mathrm{C}\right) ;{ }^{1} \mathrm{H}$ NMR $\left(500 \mathrm{MHz}, \mathrm{CDCl}_{3}\right) \delta: 7.03(\mathrm{~d}, J=16.0$ $\mathrm{Hz}, 2 \mathrm{H}, \mathrm{CH}=\mathrm{CH}), 7.39$ (d, J=8.4 Hz, 4H, ArH), 7.55 (d, $J=8.4 \mathrm{~Hz}, 4 \mathrm{H}, \mathrm{ArH}) 7.81(\mathrm{~d}, J=16.0 \mathrm{~Hz}, 2 \mathrm{H}, \mathrm{CH}=\mathrm{CH})$.

3.1 .2 中间体 $(1 E, 4 E)-1,5-$ 二(2'-吡啶基)-1,4-戊二烯3-酮 $(5 \mathbf{e})$ 的制备

(1E,4E)-1,5-二(2'-吡啶基)-1,4-戊二烯-3-酮(5e)的制 备按文献[26,39]方法进行. 收率 84\%, 黄色晶体. m.p. $148 \sim 150{ }^{\circ} \mathrm{C}$ (文献值 $145 \sim 146{ }^{\circ} \mathrm{C}$ ); ${ }^{1} \mathrm{H}$ NMR $(500$ $\left.\mathrm{MHz}, \mathrm{CDCl}_{3}\right) \delta: 7.16(\mathrm{~d}, J=16.0 \mathrm{~Hz}, 2 \mathrm{H}, \mathrm{CH}=\mathrm{CH}), 7.41$ (d, $J=6.0 \mathrm{~Hz}, 4 \mathrm{H}, \mathrm{PyH}), 7.63$ (d, $J=16.0 \mathrm{~Hz}, 2 \mathrm{H}, \mathrm{CH}=$ $\mathrm{CH}), 8.67$ (d, $J=6.0 \mathrm{~Hz}, 4 \mathrm{H}, \mathrm{PyH})$.

3.1.3中间体 $(1 E, 4 E)-1,5-$ 二(2'-氯-5-吡啶基)-1,4-戊 二烯-3-酮 $(\mathbf{5 f})$ 的制备

(1E,4E)-1,5-二(2'-氯-5-吡啶基)-1,4-戊二烯-3-酮(5f) 的制备按文献 $[26,39]$ 方法进行: 收率 69\%, 灰色晶体. m.p. $>200{ }^{\circ} \mathrm{C} ;{ }^{1} \mathrm{H}$ NMR $\left(500 \mathrm{MHz}, \mathrm{CDCl}_{3}\right) \delta: 7.10(\mathrm{~d}, J=$ $16.0 \mathrm{~Hz}, 2 \mathrm{H}, \mathrm{CH}=\mathrm{CH}), 7.41(\mathrm{~d}, J=8.3 \mathrm{~Hz}, 2 \mathrm{H}, \mathrm{PyH})$, $7.71(\mathrm{~d}, J=16.0 \mathrm{~Hz}, 2 \mathrm{H}, \mathrm{CH}=\mathrm{CH}), 7.89(\mathrm{~d}, J=8.4 \mathrm{~Hz}$, $2 \mathrm{H}, \mathrm{PyH}), 8.61$ (s, 2H, РyH).

\subsection{4中间体 1,3-二取代丙烯酮 $(\mathbf{5 h} \sim \mathbf{5 k})$ 的制备}

$15 \mathrm{~mL} 10 \% \mathrm{NaOH}$ 和 $8 \mathrm{~mL}$ 乙醇的溶液, 冰盐浴冷 却至 $-5 \sim 0{ }^{\circ} \mathrm{C}$. 滴加由醛 4 (6 mmol), 酮 MeCOY (6 $\mathrm{mmol}$ )和 $20 \mathrm{~mL}$ 乙醇组成的溶液. 继续反应 $6 \mathrm{~h}$ 至原料消 失, 过滤, 收集固体, 用水洗至中性, 粗产物经重结晶 纯化.

$E$-1-(5'-澳-2'-噻吩基)-3-(2'-氯-5'-吡啶基)丙烯酮 (5h): 收率 65\%, 黄色晶体. m.p. 188 189 ${ }^{\circ} \mathrm{C} ;{ }^{1} \mathrm{H}$ NMR $\left(500 \mathrm{MHz}, \mathrm{CDCl}_{3}\right) \delta: 7.21$ (d, J=4.1 Hz, 1H, ThH), 7.38 $(\mathrm{d}, J=15.6 \mathrm{~Hz}, 1 \mathrm{H}, \mathrm{CH}=\mathrm{CH}), 7.44(\mathrm{~d}, J=8.3 \mathrm{~Hz}, 1 \mathrm{H}$, PyH), 7.64 (d, $J=4.1 \mathrm{~Hz}, 1 \mathrm{H}, \mathrm{ThH}), 7.80$ (d, $J=15.6 \mathrm{~Hz}$, $1 \mathrm{H}, \mathrm{CH}=\mathrm{CH}), 7.93(\mathrm{dd}, J=2.5,8.5 \mathrm{~Hz}, 1 \mathrm{H}, \mathrm{PyH}), 8.65$ $(\mathrm{d}, J=2.2 \mathrm{~Hz}, 1 \mathrm{H}, \mathrm{PyH}) ;{ }^{13} \mathrm{C}$ NMR $\left(125 \mathrm{MHz}, \mathrm{CDCl}_{3}\right) \delta$ : 122.86, 124.68, 131.39, 131.54, 131.89, 132.28, 137.06, 138.07, 139.21, 148.84, 149.85, 179.99. Anal. calcd for $\mathrm{C}_{12} \mathrm{H}_{7} \mathrm{NOClBrS}$ : C 43.84, H 2.51, N 4.27; found C 43.93,

\section{H 2.48, N 4.21.}

$E$-1,3-二(5'-溴-2'-噻吩基)丙烯酩(5i): 收率 85\%, 黄 色晶体. m.p. $144 \sim 146{ }^{\circ} \mathrm{C}$ (文献值 ${ }^{[40]} 144{ }^{\circ} \mathrm{C}$ ); ${ }^{1} \mathrm{H}$ NMR $\left(500 \mathrm{MHz}, \mathrm{CDCl}_{3}\right) \delta: 6.98(\mathrm{~d}, J=15.2 \mathrm{~Hz}, 1 \mathrm{H}, \mathrm{CH}=\mathrm{CH})$, $7.06(\mathrm{~d}, J=3.8 \mathrm{~Hz}, 1 \mathrm{H}, \mathrm{ThH}), 7.10(\mathrm{~d}, J=3.8 \mathrm{~Hz}, 1 \mathrm{H}$, $\mathrm{ThH}), 7.14$ (d, $J=4.0 \mathrm{~Hz}, 1 \mathrm{H}, \mathrm{ThH}), 7.54(\mathrm{~d}, J=4.0 \mathrm{~Hz}$, $1 \mathrm{H}, \mathrm{ThH}), 7.81(\mathrm{~d}, J=15.2 \mathrm{~Hz}, 1 \mathrm{H}, \mathrm{CH}=\mathrm{CH})$.

$E$-1,3-二(4'-氯苯基)丙烯酮 $(\mathbf{5 j})$ : 收率 $88 \%$, 黄色晶 体. m.p. 161 162 ${ }^{\circ} \mathrm{C}$ (文献值 $\left.{ }^{[41]} 156 \sim 157{ }^{\circ} \mathrm{C}\right) ;{ }^{1} \mathrm{H}$ NMR $\left(500 \mathrm{MHz}, \mathrm{CDCl}_{3}\right) \delta: 7.40(\mathrm{~d}, J=8.5 \mathrm{~Hz}, 2 \mathrm{H}, \mathrm{ArH}), 7.46$ (d, $J=15.2 \mathrm{~Hz}, 1 \mathrm{H}, \mathrm{CH}=\mathrm{CH}), 7.48(\mathrm{~d}, J=8.4 \mathrm{~Hz}, 2 \mathrm{H}$, ArH), 7.58 (d, $J=8.5 \mathrm{~Hz}, 2 \mathrm{H}, \operatorname{ArH}), 7.76(\mathrm{~d}, J=15.2 \mathrm{~Hz}$, $1 \mathrm{H}, \mathrm{CH}=\mathrm{CH}), 7.96(\mathrm{~d}, J=8.5 \mathrm{~Hz}, 2 \mathrm{H}, \mathrm{ArH})$.

$E$-1-(4'-氯苯基)3-(5'-溴-2'-噻吩基)丙烯酮 $(5 \mathbf{k})$ : 收 率 $86 \%$, 黄色晶体. m.p. $133 \sim 134{ }^{\circ} \mathrm{C}$ (文献值 ${ }^{[42]} 125 \sim$ $\left.126{ }^{\circ} \mathrm{C}\right) ;{ }^{1} \mathrm{H}$ NMR $\left(500 \mathrm{MHz}, \mathrm{CDCl}_{3}\right) \delta: 7.06(\mathrm{~d}, J=3.8$ $\mathrm{Hz}, 1 \mathrm{H}, \mathrm{ThH}), 7.11$ (d, $J=3,8 \mathrm{~Hz}, 1 \mathrm{H}, \mathrm{ThH}), 7.17$ (d, $J=$ $15.3 \mathrm{~Hz}, 1 \mathrm{H}, \mathrm{CH}=\mathrm{CH}), 7.47(\mathrm{~d}, J=8.5 \mathrm{~Hz}, 2 \mathrm{H}, \mathrm{ArH})$, $7.82(\mathrm{~d}, J=15.3 \mathrm{~Hz}, 1 \mathrm{H}, \mathrm{CH}=\mathrm{CH}), 7.93(\mathrm{~d}, J=8.5 \mathrm{~Hz}$, $2 \mathrm{H}, \mathrm{ArH})$.

\subsection{5 (1E,4E)-1,5-二取代-戊二烯-3-酮腙 $(\mathbf{1 a} \sim \mathbf{1} \mathbf{p})$ 的 制备}

在含有酮 5 (5 mmol)和 2-取代肼的溴化氢 (5 mmol) 的 $30 \mathrm{~mL}$ 乙醇溶液中, 滴加 3 滴浓氢澳酸, 加热至回流, 反应 $3 \mathrm{~h}$ 后冷却至室温, 缓慢滴加饱和 $\mathrm{Na}_{2} \mathrm{CO}_{3}$ 溶液 10 $\mathrm{mL}$, 室温搅拌过夜, 加入 $10 \mathrm{~mL}$ 二氯甲烷, 分出有机相, 水相用 $10 \mathrm{~mL}$ 二氯甲烷萃取, 合并有机相, 干燥, 旋去 溶剂, 得到固体, 粗产物经重结晶纯化.

(1E,4E)-1,5-二(2'-呋喃基)-1,4-戊二烯-3-酮-(1',4', $5^{\prime}, 6$ '四氢嘧啶基)腙(1a): 收率 $90 \%$, 橙色晶体. m.p. $140 \sim 141{ }^{\circ} \mathrm{C}$ (from isopropanol); ${ }^{1} \mathrm{H}$ NMR $(500 \mathrm{MHz}$, $\left.\mathrm{CDCl}_{3}\right) \delta: 1.96\left(\mathrm{~s}, 2 \mathrm{H}, \mathrm{CH}_{2}\right), 3.36\left(\mathrm{~s}, 2 \mathrm{H}, \mathrm{NCH}_{2}\right), 3.40(\mathrm{~s}$, $2 \mathrm{H}, \mathrm{NCH}_{2}$ ), 5.20 (br s, 1H, NH), 6.31 (d, $J=3.5 \mathrm{~Hz}, 1 \mathrm{H}$, FuH), 6.39 (br s, 3H, FuH), 6.53 (s, 1H, NH), 6.88 6.96 $(\mathrm{m}, 3 \mathrm{H}, \mathrm{CH}=\mathrm{CH}), 7.38(\mathrm{~s}, 1 \mathrm{H}, \mathrm{FuH}), 7.40(\mathrm{~s}, 1 \mathrm{H}, \mathrm{FuH})$, $7.47(\mathrm{~d}, J=16.5 \mathrm{~Hz}, 1 \mathrm{H}, \mathrm{CH}=\mathrm{CH}) ;{ }^{13} \mathrm{C}$ NMR $(125 \mathrm{MHz}$, $\left.\mathrm{CDCl}_{3}\right) \delta: 21.91,38.99,39.11,108.20,109.45,111.65$, $111.74,117.17,118.73,120.56,125.37,141.89,142.50$, 146.20, 153.65, 153.92, 157.23; IR (KBr) $v_{\max }: 2883,2172$, $1655,1627,1400,1375,1110,1076,1018,966,926,744$ $\mathrm{cm}^{-1}$. Anal. calcd for $\mathrm{C}_{17} \mathrm{H}_{18} \mathrm{~N}_{4} \mathrm{O}_{2}: \mathrm{C} 65.80, \mathrm{H} 5.81, \mathrm{~N}$ 4.52; found C 65.91, H 5.79, N 4.58.

(1E,4E)-1,5-二(2'-噻吩基)-1,4-戊二烯-3-酮-(1',4', $5 ', 6$ - 四氢嘧啶基)腙(1b): 收率 $91 \%$, 黄色晶体. m.p. $188 \sim 189{ }^{\circ} \mathrm{C}$ (from methanol); ${ }^{1} \mathrm{H}$ NMR $(500 \mathrm{MHz}$, 
$\left.\mathrm{CDCl}_{3}\right) \delta: 1.99\left(\mathrm{t}, J=6.0 \mathrm{~Hz}, 2 \mathrm{H}, \mathrm{CH}_{2}\right), 3.39 \sim 3.42(\mathrm{~m}$, $\left.4 \mathrm{H}, \mathrm{NCH}_{2}\right), 6.52(\mathrm{~s}, 1 \mathrm{H}, \mathrm{NH}), 6.84(\mathrm{~d}, J=16.0 \mathrm{~Hz}, 1 \mathrm{H}$, $\mathrm{CH}=\mathrm{CH}), 6.99(\mathrm{~s}, 2 \mathrm{H}, \mathrm{ThH}), 7.02(\mathrm{~s}, 1 \mathrm{H}, \mathrm{ThH}), 7.10(\mathrm{~s}$, 1H, ThH), 7.17 (d, $J=4.5 \mathrm{~Hz}, 1 \mathrm{H}, \mathrm{ThH}), 7.20 \sim 7.23$ (m, $3 \mathrm{H}, \mathrm{ThH}, \mathrm{CH}=\mathrm{CH}), 7.39(\mathrm{~d}, J=16.0 \mathrm{~Hz}, 2 \mathrm{H}, \mathrm{CH}=\mathrm{CH})$; ${ }^{13} \mathrm{C}$ NMR (125 MHz, $\left.\mathrm{CDCl}_{3}\right) \delta: 21.89,39.01,39.06$, $120.09,122.79,124.03,125.12,125.82,126.13,126.71$, $126.91,127.59,127.60,143.38,143.57,146.43,157.14$; IR $(\mathrm{KBr}) v_{\max }: 3120,2169,1658,1633,1400,1381,1320$, 1267, 1116, 1073, 1003, 938, $698 \mathrm{~cm}^{-1}$. Anal. calcd for $\mathrm{C}_{17} \mathrm{H}_{18} \mathrm{~N}_{4} \mathrm{~S}_{2}$ : C 59.65, H 5.26, N 16.37; found C 59.75, H 5.16, N 16.37 .

(1E,4E)-1,5-二(5'-甲基-2'-噻吩基)-1,4-戊二烯-3-酮(1',4',5',6'-四氢嘧啶基)腙(1c): 收率 78\%, 黄色晶体. m.p. $205 \sim 207{ }^{\circ} \mathrm{C}$ (from chloroform); ${ }^{1} \mathrm{H}$ NMR (500 $\left.\mathrm{MHz}, \mathrm{CDCl}_{3}\right) \delta: 1.93 \sim 1.97\left(\mathrm{~m}, 2 \mathrm{H}, \mathrm{CH}_{2}\right), 2.47(\mathrm{~s}, 6 \mathrm{H}$, $\left.2 \mathrm{CH}_{3}\right), 3.35\left(\mathrm{t}, J=5.2 \mathrm{~Hz}, 2 \mathrm{H}, \mathrm{NCH}_{2}\right), 3.39(\mathrm{~d}, J=3.2 \mathrm{~Hz}$, 2H, $\left.\mathrm{NCH}_{2}\right), 5.30$ (s, 1H, NH), 6.49 (s, 1H, NH), 6.62 (s, $2 \mathrm{H}, \mathrm{ThH}), 6.71(\mathrm{~d}, J=15.9 \mathrm{~Hz}, 1 \mathrm{H}, \mathrm{CH}=\mathrm{CH}), 6.80(\mathrm{~d}$, $J=3.2 \mathrm{~Hz}, 1 \mathrm{H}, \mathrm{ThH}), 6.86$ (d, $J=3.2 \mathrm{~Hz}, 1 \mathrm{H}, \mathrm{ThH}), 7.13$ $(\mathrm{d}, J=15.9 \mathrm{~Hz}, 1 \mathrm{H}, \mathrm{CH}=\mathrm{CH}), 7.14(\mathrm{~d}, J=16.5 \mathrm{~Hz}, 1 \mathrm{H}$, $\mathrm{CH}=\mathrm{CH}), 7.28(\mathrm{~d}, J=16.0 \mathrm{~Hz}, 1 \mathrm{H}, \mathrm{CH}=\mathrm{CH}) ;{ }^{13} \mathrm{C} \mathrm{NMR}$ $\left(125 \mathrm{MHz}, \mathrm{CDCl}_{3}\right) \delta: 15.69,15.76,21.99,39.00,39.10$, $118.94,123.24,125.60,125.77,125.83,126.12,126.51$, $127.05,138.90,140.10,141.34,141.56,146.87,156.99$; IR $(\mathrm{KBr}) v_{\max }: 3381,3120,2849,2163,1624,1609$, 1536, 1436, 1403, 1387, 1313, 1295, 1120, 1070, 1009, 963, 910, $796 \mathrm{~cm}^{-1}$. Anal. calcd for $\mathrm{C}_{19} \mathrm{H}_{22} \mathrm{~N}_{4} \mathrm{~S}_{2}$ : C 61.62, H 5.95, N 15.14; found $\mathrm{C} 61.72, \mathrm{H} 6.01, \mathrm{~N} 15.24$.

$(1 E, 4 E)-1,5$-二(5'-溴-2'-噻吩基)-1,4-戊二烯-3-酮(1',4',5',6'-四氢嘧啶基)腙(1d): 收率 $80 \%$, 黄色晶体. m.p. 205 $206{ }^{\circ} \mathrm{C}$ (from methanol); ${ }^{1} \mathrm{H}$ NMR (500 MHz, $\left.\mathrm{CDCl}_{3}\right) \delta: 1.99\left(\mathrm{t}, J=5.5 \mathrm{~Hz}, 2 \mathrm{H}, \mathrm{CH}_{2}\right), 3.42(\mathrm{t}, J=6.5 \mathrm{~Hz}$, $4 \mathrm{H}, \mathrm{NCH}_{2}$ ), 5.09 (br s, 1H, NH), 6.50 (s, 1H, NH), 6.70 (d, $J=15.9 \mathrm{~Hz}, 1 \mathrm{H}, \mathrm{CH}=\mathrm{CH}), 6.75(\mathrm{~d}, J=3.7 \mathrm{~Hz}, 1 \mathrm{H}, \mathrm{ThH})$, $6.81(\mathrm{~d}, J=3.7 \mathrm{~Hz}, 1 \mathrm{H}, \mathrm{ThH}), 6.92 \sim 6.94(\mathrm{~m}, 2 \mathrm{H}, \mathrm{ThH})$, $7.06(\mathrm{~d}, J=15.8 \mathrm{~Hz}, 1 \mathrm{H}, \mathrm{CH}=\mathrm{CH}), 7.11(\mathrm{~d}, J=16.5 \mathrm{~Hz}$, $1 \mathrm{H}, \mathrm{CH}=\mathrm{CH}), 7.28(\mathrm{~d}, J=17.4 \mathrm{~Hz}, 1 \mathrm{H}, \mathrm{CH}=\mathrm{CH}) ;{ }^{13} \mathrm{C}$ NMR (125 MHz, $\left.\mathrm{CDCl}_{3}\right) \delta: 21.90,39.08,110.70,111.97$, $120.07,121.99,125.45,125.98,126.96,127.10,130.50$, $130.52,144.97,145.14,145.66,157.18$; IR (KBr) $v_{\max }$ : 2956, 2166, 1624, 1415, 1375, 1312, 1252, 1116, 1076, 1003, 970, 914, $787 \mathrm{~cm}^{-1}$. Anal. calcd for $\mathrm{C}_{17} \mathrm{H}_{16} \mathrm{~N}_{4} \mathrm{Br}_{2} \mathrm{~S}_{2}$ : C 41.71, H 4.19, N 10.89; found C 41.82, H 4.22, N 10.95.

(1E,4E)-1,5-二(5'-溴-2'-噻吩基)-1,4-戊二烯-3-酮- (1',4',5'-三氢-2-咪唑基)腙(1e)：收率 83\%，橙色晶体. m.p. 154 $155{ }^{\circ} \mathrm{C}$ (from methanol); ${ }^{1} \mathrm{H}$ NMR (500 MHz, $\left.\mathrm{CDCl}_{3}\right) \delta: 3.61\left(\mathrm{~s}, 2 \mathrm{H}, \mathrm{NCH}_{2}\right), 3.62\left(\mathrm{~s}, 2 \mathrm{H}, \mathrm{NCH}_{2}\right), 5.74(\mathrm{~s}$, br, $1 \mathrm{H}, \mathrm{NH}), 6.69$ (d, J=16.0 Hz, $1 \mathrm{H}, \mathrm{CH}=\mathrm{CH}), 6.78$ (d, $J=3.5 \mathrm{~Hz}, 1 \mathrm{H}, \mathrm{ThH}), 6.87$ (d, $J=3.5 \mathrm{~Hz}, 1 \mathrm{H}, \mathrm{ThH})$, 6.94 (t, $J=3.5 \mathrm{~Hz}, 2 \mathrm{H}, \mathrm{ThH}), 7.04$ (d, $J=16.5 \mathrm{~Hz}, 1 \mathrm{H}$, $\mathrm{CH}=\mathrm{CH}), 7.12(\mathrm{~d}, J=16.5 \mathrm{~Hz}, 1 \mathrm{H}, \mathrm{CH}=\mathrm{CH}), 7.28(\mathrm{~d}$, $J=17.0 \mathrm{~Hz}, 1 \mathrm{H}, \mathrm{CH}=\mathrm{CH}) ;{ }^{13} \mathrm{C} \mathrm{NMR}\left(125 \mathrm{MHz}, \mathrm{CDCl}_{3}\right)$ $\delta: 42.47,42.66,111.31,112.53,120.24,123.68,126.06$, $126.09,126.63,127.43,130.58,130.63,144.48,144.71$, 149.19, 165.04; IR (KBr) $v_{\max }: 2923,1618,1508,1481$, 1422, 1382, 1282, 1053, 963, $787 \mathrm{~cm}^{-1}$. Anal. calcd for $\mathrm{C}_{16} \mathrm{H}_{14} \mathrm{~N}_{4} \mathrm{Br}_{2} \mathrm{~S}_{2}$ : C 40.20, H 3.93, N 12.01; found C 40.15, H 3.90, N 11.95 .

(1E,4E)-1,5-二(5'-溴-2'- 噻吩基)-1,4-戊二烯-3-酮(1',4',5',6'- 四氢 -5',5'-二甲基-2'- 嘧啶基)腙(1f): 收率 $77 \%$, 褐色晶体. m.p. 106 $108{ }^{\circ} \mathrm{C}$ (from methanol); ${ }^{1} \mathrm{H}$ NMR (500 MHz, $\left.\mathrm{CDCl}_{3}\right) \delta: 1.11\left(\mathrm{~s}, 6 \mathrm{H}, 2 \mathrm{CH}_{3}\right), 3.06(\mathrm{~s}$, $\left.2 \mathrm{H}, \mathrm{NCH}_{2}\right), 3.08$ (s, 2H, $\left.\mathrm{NCH}_{2}\right), 6.52$ (s, 1H, NH), 6.70 (d, $J=16.0 \mathrm{~Hz}, 1 \mathrm{H}, \mathrm{CH}=\mathrm{CH}), 6.75(\mathrm{~d}, J=2.1 \mathrm{~Hz}, 1 \mathrm{H}, \mathrm{ThH})$, $6.82(\mathrm{~d}, J=2.4 \mathrm{~Hz}, 1 \mathrm{H}, \mathrm{ThH}), 6.93(\mathrm{t}, J=2.4 \mathrm{~Hz}, 2 \mathrm{H}$, $\mathrm{ThH}), 7.04$ (d, $J=15.5 \mathrm{~Hz}, 1 \mathrm{H}, \mathrm{CH}=\mathrm{CH}), 7.10(\mathrm{~d}, J=$ $17.0 \mathrm{~Hz}, 1 \mathrm{H}, \mathrm{CH}=\mathrm{CH}), 7.28(\mathrm{~d}, J=19.0 \mathrm{~Hz}, 1 \mathrm{H}, \mathrm{CH}=$ $\mathrm{CH}) ;{ }^{13} \mathrm{C}$ NMR $\left(125 \mathrm{MHz}, \mathrm{CDCl}_{3}\right) \delta: 24.43,28.68,50.80$, $110.79,112.06,119.97,122.24,125.70,126.06,127.05$, $130.51,144.91,145.08,145.85,156.20$; IR (KBr) $v_{\max }$ : 2950, 2920, 1594, 1418, 1392, 1365, 1286, 1262, 1096, 1053, 963, $791 \mathrm{~cm}^{-1}$. Anal. calcd for $\mathrm{C}_{19} \mathrm{H}_{20} \mathrm{~N}_{4} \mathrm{Br}_{2} \mathrm{~S}_{2}$ : C 43.82, H 4.41, N 9.46; found C 43.79, H 4.40, N 9.49.

$(1 E, 4 E)-1,5-$ 二 (2'- 吡啶基 )-1,4-戊二烯 -3-酮 $\left(1^{\prime}, 4^{\prime}, 5^{\prime}, 6^{\prime}\right.$-四氢嘧啶基)腙(1g): 收率 $72 \%$, 土黄色晶体. m.p. 252 253 ${ }^{\circ} \mathrm{C}$ (from methanol); ${ }^{1} \mathrm{H}$ NMR (500 MHz, $\left.\mathrm{CDCl}_{3}\right) \delta: 2.01\left(\mathrm{t}, J=6.0 \mathrm{~Hz}, 2 \mathrm{H}, \mathrm{CH}_{2}\right), 3.41(\mathrm{~s}, 2 \mathrm{H}$, $\mathrm{NCH}_{2}$ ), 3.44 (s, 2H, $\mathrm{NCH}_{2}$ ), 5.45 (br s, 1H, NH), 6.60 (s, $1 \mathrm{H}, \mathrm{NH}), 6.97(\mathrm{~d}, J=16.0 \mathrm{~Hz}, 1 \mathrm{H}, \mathrm{CH}=\mathrm{CH}), 7.02(\mathrm{~d}, J=$ $17.0 \mathrm{~Hz}, 1 \mathrm{H}, \mathrm{CH}=\mathrm{CH}), 7.23(\mathrm{~d}, J=16.0 \mathrm{~Hz}, 1 \mathrm{H}, \mathrm{CH}=$ $\mathrm{CH}), 7.33$ (d, $J=6.0 \mathrm{~Hz}, 2 \mathrm{H}, \mathrm{PyH}), 7.40$ (d, $J=6.0 \mathrm{~Hz}$, $2 \mathrm{H}, \mathrm{PyH}), 7.81(\mathrm{~d}, J=17.0 \mathrm{~Hz}, 1 \mathrm{H}, \mathrm{CH}=\mathrm{CH}), 8.55 \sim 8.58$ $(\mathrm{m}, 4 \mathrm{H}, \mathrm{PyH}) ;{ }^{13} \mathrm{C} \mathrm{NMR}\left(125 \mathrm{MHz}, \mathrm{CDCl}_{3}\right) \delta: 18.12$, $42.51,42.65,120.94,121.28,124.51,128.70,130.75$, $131.17,144.53,144.63,149.67,150.20,165.11$; IR (KBr) $v_{\max }: 3226,2359,1582,1419,1366,1260,1230,1117$, 1097, 994, 964, 921, $808 \mathrm{~cm}^{-1}$. Anal. calcd for $\mathrm{C}_{19} \mathrm{H}_{20} \mathrm{~N}_{6}$ : C 67.32, H 6.12, N 24.70; found C 67.40, H 6.06, N 24.75 . 
(1E,4E)-1,5-二(2'-氯-5'-吡啶基)-1,4-戊二烯-3-酮(1',4',5',6'-四氢嘧啶基)腙(1h): 收率 $80 \%$, 黄色晶体. m.p. $240 \sim 242{ }^{\circ} \mathrm{C}$ (from methanol); ${ }^{1} \mathrm{H}$ NMR (500 MHz, $\left.\mathrm{CDCl}_{3}\right) \delta: 1.99 \sim 2.02\left(\mathrm{~m}, 2 \mathrm{H}, \mathrm{CH}_{2}\right), 3.41\left(\mathrm{~s}, 2 \mathrm{H}, \mathrm{NCH}_{2}\right)$, $3.44\left(\mathrm{~s}, 2 \mathrm{H}, \mathrm{NCH}_{2}\right), 5.60(\mathrm{~s}, 1 \mathrm{H}, \mathrm{NH}), 6.7(\mathrm{~s}, 1 \mathrm{H}, \mathrm{NH})$, $6.98 \sim 7.08(\mathrm{~m}, 3 \mathrm{H}, \mathrm{CH}=\mathrm{CH}), 7.29 \sim 7.31(\mathrm{~m}, 2 \mathrm{H}, \mathrm{PyH})$, $7.66(\mathrm{~d}, J=16.8 \mathrm{~Hz}, 1 \mathrm{H}, \mathrm{CH}=\mathrm{CH}), 7.76 \sim 7.79(\mathrm{~m}, 1 \mathrm{H}$, PyH), $7.88 \sim 7.90$ (m, 1H, РyH), $8.44 \sim 8.46(\mathrm{~m}, 2 \mathrm{H}, \mathrm{PyH})$; ${ }^{13} \mathrm{C}$ NMR $\left(125 \mathrm{MHz}, \mathrm{CDCl}_{3}\right) \delta: 21.89,39.22,122.87$, $124.18,124.20,124.29,127.65,129.55,132.32,132.45$, $135.19,135.60,145.77,148.15,148.60,149.63,150.26$, 157.38; IR (KBr) $v_{\max }: 3387,3042,2964,1609,1506$, 1461, 1371, 1198, 911, 824, $719 \mathrm{~cm}^{-1}$. Anal. calcd for $\mathrm{C}_{19} \mathrm{H}_{18} \mathrm{C}_{12} \mathrm{~N}_{6}$ : C 56.87, H 4.52, N 20.94; found C 56.99, H 4.53, N 21.01.

(1E,4E)-1,5-二 (4'- 氯苯基 )-1,4-戊二烯 -3- 酮 (1',4',5',6'-四氢嘧啶基)腙(1i): 收率 89\%, 黄色晶体. m.p. 198 $200{ }^{\circ} \mathrm{C}$ (from ethanol); ${ }^{1} \mathrm{H}$ NMR $\left(500 \mathrm{MHz}, \mathrm{CDCl}_{3}\right.$ ) $\delta: 1.84 \sim 1.88\left(\mathrm{~m}, 2 \mathrm{H}, \mathrm{CH}_{2}\right), 3.26\left(\mathrm{~s}, 2 \mathrm{H}, \mathrm{NCH}_{2}\right), 3.35(\mathrm{~s}$, $\left.2 \mathrm{H}, \mathrm{NCH}_{2}\right), 6.04(\mathrm{~s}, 1 \mathrm{H}, \mathrm{NH}), 6.56(\mathrm{~s}, 1 \mathrm{H}, \mathrm{NH}), 7.00 \sim$ $7.07(\mathrm{~m}, 3 \mathrm{H}, \mathrm{HC}=\mathrm{CH}), 7.26 \sim 7.30(\mathrm{~m}, 4 \mathrm{H}, \mathrm{ArH}), 7.41(\mathrm{~d}$, $J=8.5 \mathrm{~Hz}, 2 \mathrm{H}, \mathrm{ArH}), 7.46(\mathrm{~d}, J=8.5 \mathrm{~Hz}, 2 \mathrm{H}, \mathrm{ArH}), 7.64$ $(\mathrm{d}, J=16.8 \mathrm{~Hz}, 1 \mathrm{H}, \mathrm{HC}=\mathrm{CH}) ;{ }^{13} \mathrm{C}$ NMR $(125 \mathrm{MHz}$, $\left.\mathrm{CDCl}_{3}\right) \delta: 21.89,39.03,121.30,127.66,128.12,128.57$, $128.78,131.59,132.86,133.47,136.12,136.33,147.04$, 157.23; IR (KBr) $v_{\max }: 3394,3171,2972,1641,1592$, 1541, 1492, 1373, 1120, 965, 818, $696 \mathrm{~cm}^{-1}$. Anal. calcd for $\mathrm{C}_{21} \mathrm{H}_{20} \mathrm{Cl}_{2} \mathrm{~N}_{4}$ : C 63.17, H 5.01, N 14.03; found C 63.43, H 5.02, N 14.10 .

$E$-1-(5'-溴-2'-噻吩基)-3-(2'-氯-5'-吡啶基)丙烯酮(1',4',5'-三氢-2-咪唑基)腙 $(\mathbf{1 j})$ : 收率 70\%, 橙色晶体. m.p. $198 \sim 199{ }^{\circ} \mathrm{C}$ (from ethanol); ${ }^{1} \mathrm{H}$ NMR $(500 \mathrm{MHz}$, $\left.\mathrm{CDCl}_{3}\right) \delta: 3.43\left(\mathrm{t}, J=5.6 \mathrm{~Hz}, 4 \mathrm{H}, \mathrm{NCH}_{2}\right), 5.10(\mathrm{~s}, 1 \mathrm{H}$, $\mathrm{NH}), 6.55$ (s, 1H, NH), 7.02 (d, $J=4.4 \mathrm{~Hz}, 1 \mathrm{H}, \mathrm{ThH}), 7.15$ $(\mathrm{d}, J=16.0 \mathrm{~Hz}, 1 \mathrm{H}, \mathrm{CH}=\mathrm{CH}), 7.20(\mathrm{~d}, J=4.4 \mathrm{~Hz}, 1 \mathrm{H}$, ThH), $7.21(\mathrm{~d}, J=16.0 \mathrm{~Hz}, 1 \mathrm{H}, \mathrm{CH}=\mathrm{CH}), 7.28(\mathrm{~d}, J=8.4$ $\mathrm{Hz}, 1 \mathrm{H}, \mathrm{PyH}), 7.78$ (dd, $J=2.4,8.4 \mathrm{~Hz}, 1 \mathrm{H}, \mathrm{PyH}), 8.43$ (d, $J=2.4 \mathrm{~Hz}, 1 \mathrm{H}, \mathrm{PyH}) ;{ }^{13} \mathrm{C} \mathrm{NMR}\left(125 \mathrm{MHz}, \mathrm{CDCl}_{3}\right) \delta$ : $39.23,117.21,124.17,124.78,125.44,127.48,128.06$, 132.34, 135.30, 136.08, 140.47, 148.32, 149.72, 156.89; IR (KBr) $v_{\max }: 3396,2957,2862,1596,1571,1460,1378$, 1264, 1128, 964, $768 \mathrm{~cm}^{-1}$. Anal. calcd for $\mathrm{C}_{15} \mathrm{H}_{13} \mathrm{Br}-$ $\mathrm{ClN}_{5} \mathrm{~S}$ : C 43.86, H 3.19, N 17.05; found C 43.99, H 3.20, $\mathrm{N} 16.99$.

$E$-1-(5'-澳-2'-噻吩基)-3-(2'-氯-5'-吡啶基)丙烯酮- (1',4',5',6'-四氢嘧啶基)腙(1k): 收率 75\%, 黄色晶体. m.p. 205 206 ${ }^{\circ} \mathrm{C}$ (from methanol); ${ }^{1} \mathrm{H}$ NMR $(500 \mathrm{MHz}$, $\left.\mathrm{CDCl}_{3}\right) \delta: 2.02 \sim 2.07\left(\mathrm{~m}, 2 \mathrm{H}, \mathrm{CH}_{2}\right), 3.46(\mathrm{t}, J=5.2 \mathrm{~Hz}$, $\left.4 \mathrm{H}, \mathrm{NCH}_{2}\right), 5.02(\mathrm{~s}, 1 \mathrm{H}, \mathrm{NH}), 6.52(\mathrm{~s}, 1 \mathrm{H}, \mathrm{NH}), 7.02(\mathrm{~d}$, $J=4.2 \mathrm{~Hz}, 1 \mathrm{H}, \mathrm{ThH}), 7.15(\mathrm{~d}, J=16.0 \mathrm{~Hz}, 1 \mathrm{H}, \mathrm{CH}=\mathrm{CH})$, 7.20 (d, $J=3.9 \mathrm{~Hz}, 1 \mathrm{H}, \mathrm{ThH}), 7.22$ (d, $J=15.7 \mathrm{~Hz}, 1 \mathrm{H}$, $\mathrm{CH}=\mathrm{CH}), 7.29(\mathrm{~d}, J=8.3 \mathrm{~Hz}, 1 \mathrm{H}, \mathrm{PyH}), 7.78(\mathrm{dd}, J=2.3$, $8.3 \mathrm{~Hz}, 1 \mathrm{H}, \mathrm{PyH}), 8.45$ (d, $J=2.2 \mathrm{~Hz}, 1 \mathrm{H}, \mathrm{PyH}) ;{ }^{13} \mathrm{C} \mathrm{NMR}$ $\left(125 \mathrm{MHz}, \mathrm{CDCl}_{3}\right) \delta: 21.90,39.22,117.24,124.18$, 124.81, 125.46, 127.47, 128.08, 132.34, 135.29, 136.08, $140.53,148.32,149.74,156.89$; IR (KBr) $v_{\max }: 3396,2957$, 2862, 1597, 1572, 1460, 1308, 1264, 1128, 964, $768 \mathrm{~cm}^{-1}$. Anal. calcd for $\mathrm{C}_{16} \mathrm{H}_{15} \mathrm{BrClN}_{5} \mathrm{~S}$ : C 45.24, H 3.56, N 16.49; found C 45.19, H 3.55, N 16.41.

E-1-(5'-澳-2'-噻吩基)-3-(2'-氯-5'-吡啶基)丙烯酮(1',4',5',6'-四氢-5',5'-二甲基-2'-嘧啶基)腙 $(\mathbf{1 m})$ : 收率 $80 \%$, 橘黄色晶体, m.p. 189 191 ${ }^{\circ} \mathrm{C}$ (from methanol); ${ }^{1} \mathrm{H}$ NMR (500 MHz, $\left.\mathrm{CDCl}_{3}\right) \delta: 1.12\left(\mathrm{~s}, 6 \mathrm{H}, \mathrm{CH}_{3}\right), 3.10$ (s, $\left.4 \mathrm{H}, \mathrm{NCH}_{2}\right), 5.07(\mathrm{~s}, 1 \mathrm{H}, \mathrm{NH}), 6.55(\mathrm{~s}, 1 \mathrm{H}, \mathrm{NH}), 7.03(\mathrm{~d}$, $J=4.2 \mathrm{~Hz}, 1 \mathrm{H}, \mathrm{ThH}), 7.16(\mathrm{~d}, J=16.0 \mathrm{~Hz}, 1 \mathrm{H}, \mathrm{CH}=\mathrm{CH})$, 7.20 (d, $J=4.2 \mathrm{~Hz}, 1 \mathrm{H}, \mathrm{ThH}), 7.22(\mathrm{~d}, J=16.0 \mathrm{~Hz}, 1 \mathrm{H}$, $\mathrm{CH}=\mathrm{CH}), 7.29(\mathrm{~d}, J=8.4 \mathrm{~Hz}, 1 \mathrm{H}, \mathrm{PyH}), 7.79(\mathrm{dd}, J=2.5$, $8.4 \mathrm{~Hz}, 1 \mathrm{H}, \mathrm{PyH}), 8.44$ (d, $J=2.5 \mathrm{~Hz}, 1 \mathrm{H}, \mathrm{PyH}) ;{ }^{13} \mathrm{C}$ NMR $\left(125 \mathrm{MHz}, \mathrm{CDCl}_{3}\right) \delta: 24.48,28.83,50.87,50.93,117.23$, $124.18,124.82,125.47,127.46,128.15,132.36,135.28$, $136.06,140.46,148.33,149.74,156.13$; IR (KBr) $v_{\max }$ : 3396, 2957, 2862, 1571, 1460, 1307, 1265, 1128, 1021, $964,768 \mathrm{~cm}^{-1}$. Anal. calcd for $\mathrm{C}_{18} \mathrm{H}_{19} \mathrm{BrClN}_{5} \mathrm{~S}: \mathrm{C} 47.75, \mathrm{H}$ 4.23, N 15.47; found C 47.72, H 4.21, N 15.52.

$E-1,3$-二(5'-溴-2'-噻吩基)丙烯酮-(1',4',5',6'-四氢嘧 啶基)腙(1n): 收率 $90 \%$, 淡黄色晶体. m.p. $158 \sim 160{ }^{\circ} \mathrm{C}$ (from ethanol); ${ }^{1} \mathrm{H}$ NMR $\left(500 \mathrm{MHz}, \mathrm{CDCl}_{3}\right) \delta: 3.60 \sim 3.67$ (m, 4H, $\left.\mathrm{NCH}_{2}\right), 4.70(\mathrm{~s}, 1 \mathrm{H}, \mathrm{NH}), 5.83(\mathrm{~s}, 1 \mathrm{H}, \mathrm{NH}), 6.78$ (d, $J=3.8 \mathrm{~Hz}, 1 \mathrm{H}, \mathrm{ThH}), 6.88(\mathrm{~d}, J=15.6 \mathrm{~Hz}, 1 \mathrm{H}, \mathrm{CH}=$ $\mathrm{CH}), 6.94(\mathrm{~d}, J=3.8 \mathrm{~Hz}, 1 \mathrm{H}, \mathrm{ThH}), 7.02(\mathrm{~d}, J=4.2 \mathrm{~Hz}$, 1H, ThH), 7.18 (d, $J=4.2 \mathrm{~Hz}, 1 \mathrm{H}, \mathrm{ThH}), 7.25$ (d, $J=15.6$ $\mathrm{Hz}, 1 \mathrm{H}, \mathrm{CH}=\mathrm{CH}) ;{ }^{13} \mathrm{C} \mathrm{NMR}\left(125 \mathrm{MHz}, \mathrm{CDCl}_{3}\right) \delta: 42.51$, $42.69,111.51,118.16,124.23,124.92,126.72,126.94$, 127.62, 130.57, 135.78, 144.37, 164.03; IR (KBr) $v_{\max }$ : 3270, 2968, 1657, 1642, 1612, 1576, 1490, 1421, 1053, $963,806 \mathrm{~cm}^{-1}$. Anal. calcd for $\mathrm{C}_{14} \mathrm{H}_{12} \mathrm{Br}_{2} \mathrm{~N}_{4} \mathrm{~S}_{2}: \mathrm{C} 36.54, \mathrm{H}$ 2.61, N 12.18; found C 36.46, H 2.62, N 12.17 .

$E$-1,3-二(5'-溴-2'-噻吩基)丙烯酮-(1',4',5',6'-四氢嘧 啶基)腙(10): 收率 68\%, 黄色晶体. m.p. 179 181 ${ }^{\circ} \mathrm{C}$ (from ethanol); ${ }^{1} \mathrm{H}$ NMR (500 MHz, $\mathrm{CDCl}_{3}$ ) $\delta: 1.99 \sim 2.03$ 
(m, 2H, $\mathrm{CH}_{2}$ ), 3.42 (s, 4H, NCH$), 5.03$ (s, 1H, NH), 6.53 (s, 1H, NH), 6.78 (d, $J=5.0 \mathrm{~Hz}, 1 \mathrm{H}, \mathrm{ThH}), 6.91$ (d, $J=$ $15.7 \mathrm{~Hz}, 1 \mathrm{H}, \mathrm{CH}=\mathrm{CH}), 6.93(\mathrm{~d}, J=3.8 \mathrm{~Hz}, 1 \mathrm{H}, \mathrm{ThH})$, $7.02(\mathrm{~d}, J=3.8 \mathrm{~Hz}, 1 \mathrm{H}, \mathrm{ThH}), 7.18(\mathrm{~d}, J=4.1 \mathrm{~Hz}, 1 \mathrm{H}$, $\mathrm{ThH}), 7.24(\mathrm{~d}, J=15.6 \mathrm{~Hz}, 1 \mathrm{H}, \mathrm{CH}=\mathrm{CH}) ;{ }^{13} \mathrm{C}$ NMR $(125$ $\left.\mathrm{MHz}, \mathrm{CDCl}_{3}\right) \delta: 21.96,39.10,39.28,110.95,117.15$, $122.66,125.28,125.56,126.37,127.46,130.51,136.09$, 140.72, 144.78, 156.68; IR (KBr) $v_{\max }: 3431,3373,2865$, 1591, 1564, 1376, 1317, 1261, 1116, 960, $793 \mathrm{~cm}^{-1}$. Anal. calcd for $\mathrm{C}_{15} \mathrm{H}_{14} \mathrm{Br}_{2} \mathrm{~N}_{4} \mathrm{~S}_{2}$ : C 37.99, H 2.98, N 11.81; found C 38.07, H 2.97, N 11.70.

$E-1,3$-二(5'-溴-2'- 噻吩基)丙烯酮-( $1^{\prime}, 4^{\prime}, 5^{\prime}, 6^{\prime}$ - 四氢5',5'-二甲基-2'-嘧啶基)腙(1p): 收率 59\%, 黄色晶体. m.p. $188 \sim 190{ }^{\circ} \mathrm{C}$ (from ethanol); ${ }^{1} \mathrm{H}$ NMR $(500 \mathrm{MHz}$, $\left.\mathrm{CDCl}_{3}\right) \delta: 1.10$ (s, $\left.6 \mathrm{H}, \mathrm{CH}_{3}\right), 3.06$ (s, $\left.2 \mathrm{H}, \mathrm{NCH}_{2}\right), 3.07$ (s, $\left.2 \mathrm{H}, \mathrm{NCH}_{2}\right), 5.06(\mathrm{~s}, 1 \mathrm{H}, \mathrm{NH}), 6.54(\mathrm{~s}, 1 \mathrm{H}, \mathrm{NH}), 6.77(\mathrm{~d}$, $J=4.0 \mathrm{~Hz}, 1 \mathrm{H}, \mathrm{ThH}), 6.91(\mathrm{~d}, J=15.0 \mathrm{~Hz}, 1 \mathrm{H}, \mathrm{CH}=\mathrm{CH})$, $6.93(\mathrm{~d}, J=4.0 \mathrm{~Hz}, 1 \mathrm{H}, \mathrm{ThH}), 7.01$ (d, $J=4.0 \mathrm{~Hz}, 1 \mathrm{H}$, ThH), 7.18 (d, $J=4.0 \mathrm{~Hz}, 1 \mathrm{H}, \mathrm{ThH}), 7.24$ (d, $J=15.0 \mathrm{~Hz}$, $1 \mathrm{H}, \mathrm{CH}=\mathrm{CH}) ;{ }^{13} \mathrm{C}$ NMR $\left(125 \mathrm{MHz}, \mathrm{CDCl}_{3}\right) \delta: 24.48$, $28.83,50.87,50.93,110.92,117.10,122.67,125.34$, $125.57,126.35,127.46,130.52,136.08,140.59,144.81$, 155.92; IR (KBr) $v_{\max }: 3431,3373,2963,1591,1564$, 1377, 1317, 1117, 1016, 960, $793 \mathrm{~cm}^{-1}$. Anal. calcd for $\mathrm{C}_{17} \mathrm{H}_{18} \mathrm{Br}_{2} \mathrm{~N}_{4} \mathrm{~S}_{2}$ : C 40.64, H 3.62, N 11.16; found C 40.67, $\mathrm{H} 3.67, \mathrm{~N} 11.10$.

\subsection{6中间体环氧化合物 6 的制备}

在 $20 \mathrm{~mL}$ 丙酮中加入硫酸二甲酯 $(8.6 \mathrm{mmol})$ 和二甲 硫醚 $(8.6 \mathrm{mmol})$, 室温搅拌 $5 \mathrm{~h}$ 后, 蒸除溶剂得白色固体. 将此白色固体溶于 $60 \mathrm{~mL}$ 二氯甲烷后加叔丁醇钾 $(10.8$ $\mathrm{mmol})$, 室温搅拌 $5 \mathrm{~min}$ 后, 加入酮 5 (7.2 $\mathrm{mmol})$, 在室 温下反应 $3 \mathrm{~h}$. 加入 $50 \mathrm{~mL}$ 水, 分出有机层, 并用水洗 3 次 $(20 \mathrm{~mL} \times 3)$ 后, 用无水碳酸钾干燥, 蒸除溶剂后得淡 黄色稠状液体, 粗产率约 $99 \%{ }^{[35]}$. 未经分离, 直接用于 下步反应.

\subsection{7 化合物 $\mathbf{2 a} \sim \mathbf{2 e}$ 的的制备}

$50 \mathrm{~mL}$ 的 $N, N$-二甲基甲酰胺(DMF)中加入在 3.1 .6 节中制备的环氧化合物 $6(7.2 \mathrm{mmol})$ 、胺 $(8.6 \mathrm{mmol})$ 和氢 氧化钠 $(8.6 \mathrm{mmol}), 50{ }^{\circ} \mathrm{C}$ 反应 $3 \mathrm{~h}$. 减压蒸除 DMF 后加入 $50 \mathrm{~mL}$ 的乙醚搅拌至溶, 并用饱和食盐水洗 $(30 \mathrm{~mL} \times 3)$, 有机层经干燥后蒸除乙醚. 用 $V$ (二氯甲烷 $): V$ (甲醇 $)=$ $80: 1$ 的洗脱剂柱色谱纯化.

$E$-2,4-二(4'-氯苯基)-1-2'-(1',4',5',6'-四氢嘧啶基)氨 基丁烯-2-醇(2a): 收率 68\%, 白色晶体. m.p. 190 $191{ }^{\circ} \mathrm{C}$ (from ethanol); ${ }^{1} \mathrm{H}$ NMR $\left[500 \mathrm{MHz},\left(\mathrm{CD}_{3}\right)_{2} \mathrm{SO}\right] \delta$ : $1.65\left(\mathrm{t}, J=5.2 \mathrm{~Hz}, 2 \mathrm{H}, \mathrm{CH}_{2}\right), 3.05(\mathrm{~d}, J=5.2 \mathrm{~Hz}, 2 \mathrm{H}$, $\left.\mathrm{NCH}_{2}\right), 3.08 \sim 3.10\left(\mathrm{~m}, 1 \mathrm{H}, \mathrm{NCH}_{2}\right), 3.23 \sim 3.26(\mathrm{~m}, 1 \mathrm{H}$, $\left.\mathrm{NCH}_{2}\right), 3.63\left[\mathrm{~d},{ }^{2} J=15.1 \mathrm{~Hz}, 1 \mathrm{H}, \mathrm{C}(\mathrm{OH}) \mathrm{CH}\right], 3.71\left[\mathrm{~d},{ }^{2} J\right.$ $=15.1 \mathrm{~Hz}, 1 \mathrm{H}, \mathrm{C}(\mathrm{OH}) \mathrm{CH}], 6.71(\mathrm{~d}, J=15.9 \mathrm{~Hz}, 1 \mathrm{H}, \mathrm{CH}$ $=\mathrm{CH}), 6.87(\mathrm{~d}, J=15.9 \mathrm{~Hz}, 1 \mathrm{H}, \mathrm{CH}=\mathrm{CH}), 7.39(\mathrm{~d}, J=$ $8.4 \mathrm{~Hz}, 2 \mathrm{H}, \mathrm{ArH}), 7.44$ (d, $J=8.6 \mathrm{~Hz}, 2 \mathrm{H}, \mathrm{ArH}), 7.49$ (d, $J$ $=8.4 \mathrm{~Hz}, 2 \mathrm{H}, \mathrm{ArH}), 7.67(\mathrm{~d}, J=8.6 \mathrm{~Hz}, 2 \mathrm{H}, \mathrm{ArH}), 8.41(\mathrm{~s}$, $1 \mathrm{H}, \mathrm{NH}) ;{ }^{13} \mathrm{C}$ NMR $\left[125 \mathrm{MHz},\left(\mathrm{CD}_{3}\right)_{2} \mathrm{SO}\right] \delta: 20.27,37.57$, $47.98,59.61,77.05,127.52,127.82,128.07,128.52$, 131.81, 131.93, 133.72, 135.41, 142.76, 155.20, 167.27; IR $(\mathrm{KBr}) v_{\max }: 3153,1658,1642,1620,1612,1491,1402$, 1092, 1003, 810, $623 \mathrm{~cm}^{-1}$. HRMS (ESI) calcd for $\mathrm{C}_{20} \mathrm{H}_{22} \mathrm{Cl}_{2} \mathrm{~N}_{3} \mathrm{O}[\mathrm{M}+\mathrm{H}]^{+}: 390.1134$, found 390.1158 .

$E$-2,4-二(4'-氯苯基)-1-(2'-嘧啶氨基)丁烯-2-醇(2b): 收率 $83 \%$, 白色晶体. m.p. $151 \sim 152{ }^{\circ} \mathrm{C}$ (from ethanol); ${ }^{1} \mathrm{H}$ NMR $\left(500 \mathrm{MHz}, \mathrm{CDCl}_{3}\right) \delta: 3.86(\mathrm{dd}, J=6.3,14.6 \mathrm{~Hz}$, $1 \mathrm{H}, \mathrm{NCH}), 3.95$ (dd, $J=6.9,14.6 \mathrm{~Hz}, 1 \mathrm{H}, \mathrm{NCH}), 5.44$ (s, br, $1 \mathrm{H}, \mathrm{NH}), 5.86(\mathrm{~s}, 1 \mathrm{H}, \mathrm{OH}), 6.50(\mathrm{~d}, J=15.8 \mathrm{~Hz}, 1 \mathrm{H}$, $\mathrm{CH}=\mathrm{CH}), 6.62(\mathrm{~d}, J=4.9 \mathrm{~Hz}, 1 \mathrm{H}, \mathrm{PyH}), 6.76(\mathrm{~d}, J=15.8$ $\mathrm{Hz}, 1 \mathrm{H}, \mathrm{CH}=\mathrm{CH}), 7.26(\mathrm{~d}, J=8.7 \mathrm{~Hz}, 2 \mathrm{H}, \mathrm{ArH}), 7.30(\mathrm{~d}$, $J=8.7 \mathrm{~Hz}, 2 \mathrm{H}, \mathrm{ArH}), 7.34$ (d, $J=8.6 \mathrm{~Hz}, 2 \mathrm{H}, \mathrm{ArH}), 7.50$ (d, $J=8.6 \mathrm{~Hz}, 2 \mathrm{H}, \mathrm{ArH}), 8.28$ (d, $J=4.9 \mathrm{~Hz}, 2 \mathrm{H}, \mathrm{PyH}$ ); ${ }^{13} \mathrm{C}$ NMR $\left(125 \mathrm{MHz}, \mathrm{CDCl}_{3}\right) \delta: 52.71,77.98,111.43$, $127.17,127.80,128.47,128.66,128.71,132.96,133.27$, 133.63, 135.14, 142.87, 157.95, 162.80; IR (KBr) $v_{\max }$ : 3343, 1641, 1604, 1547, 1492, 1402, 1336, 1092, 800, 740 $\mathrm{cm}^{-1}$. Anal. calcd for $\mathrm{C}_{20} \mathrm{H}_{17} \mathrm{Cl}_{2} \mathrm{~N}_{3} \mathrm{O}$ : C $62.19, \mathrm{H} 4.44, \mathrm{~N}$ 10.88; found C 62.07, H 4.42, N 10.72.

$E-2,4$-二(4'-氯苯基)-1-(1',4'-二氮杂苯-2'-氨基)丁烯2-醇(2c)：收率 81\%，白色晶体. m.p. 156 $157{ }^{\circ} \mathrm{C}$ (from ethanol). ${ }^{1} \mathrm{H}$ NMR (500 MHz, $\mathrm{CDCl}_{3}$ ) $\delta: 3.78$ (dd, $J=6.0$ $\mathrm{Hz}, 14.3 \mathrm{~Hz}, 1 \mathrm{H}, \mathrm{NCH}), 3.88$ (dd, $J=6.1,14.3 \mathrm{~Hz}, 1 \mathrm{H}$, $\mathrm{NCH}), 5.10$ (t, $J=5.9 \mathrm{~Hz}, 1 \mathrm{H}, \mathrm{NH}), 5.66$ (s, 1H, OH), 6.45 (d, $J=15.8 \mathrm{~Hz}, 1 \mathrm{H}, \mathrm{CH}=\mathrm{CH}), 6.71(\mathrm{~d}, J=15.8 \mathrm{~Hz}, 1 \mathrm{H}$, $\mathbf{C H}=\mathrm{CH}), 7.22(\mathrm{~d}, J=9.3 \mathrm{~Hz}, 2 \mathrm{H}, \operatorname{ArH}), 7.25(\mathrm{~d}, J=9.3$ $\mathrm{Hz}, 2 \mathrm{H}, \mathrm{ArH}), 7.30$ (d, $J=8.6 \mathrm{~Hz}, 2 \mathrm{H}, \operatorname{ArH}), 7.46$ (d, $J=$ $8.6 \mathrm{~Hz}, 2 \mathrm{H}, \mathrm{ArH}), 7.77$ (d, $J=2.8 \mathrm{~Hz}, 1 \mathrm{H}, \mathrm{PyH}), 7.80$ (d, $J=2.8 \mathrm{~Hz}, 1 \mathrm{H}, \mathrm{PyH}), 7.90$ (s, 1H, PyH); ${ }^{13} \mathrm{C}$ NMR $(125$ $\left.\mathrm{MHz}, \mathrm{CDCl}_{3}\right) \delta: 52.64,77.69,127.18,127.84,128.59$, $128.76,128.92,133.16,133.42,133.47,133.87,134.98$, 140.53, 142.68, 154.57; IR (KBr) $v_{\max }: 3366,1659,1594$, 1402, 1093, 1003, 807, $624 \mathrm{~cm}^{-1}$. Anal. calcd for $\mathrm{C}_{20} \mathrm{H}_{17} \mathrm{Cl}_{2} \mathrm{~N}_{3} \mathrm{O}$ : C 62.19, H 4.44, N 10.88; found C 62.10, $\mathrm{H} 4.40, \mathrm{~N} 10.87$.

( $1 E, 4 E$ )-1,5-二(4'-氯苯基)-3-(2'-嘧啶氨基甲基)-1,4- 
丁二烯-3-醇 (2d): 收率 45\%, 白色晶体. m.p. 118 $120{ }^{\circ} \mathrm{C}$ (from ethanol); ${ }^{1} \mathrm{H}$ NMR $\left(500 \mathrm{MHz}, \mathrm{CDCl}_{3}\right) \delta$ : $3.71(\mathrm{~d}, J=6.3 \mathrm{~Hz}, 2 \mathrm{H}, \mathrm{NCH}), 5.41(\mathrm{br} \mathrm{s}, 1 \mathrm{H}, \mathrm{OH}), 5.66$ $(\mathrm{t}, J=6.1 \mathrm{~Hz}, 1 \mathrm{H}, \mathrm{NH}), 6.31(\mathrm{~d}, J=15.8 \mathrm{~Hz}, 2 \mathrm{H}, \mathrm{CH}=$ $\mathrm{CH}), 6.59$ (t, $J=4.8 \mathrm{~Hz}, 1 \mathrm{H}, \mathrm{PyH}), 6.76(\mathrm{~d}, J=15.8 \mathrm{~Hz}$, $2 \mathrm{H}, \mathrm{CH}=\mathrm{CH}$ ), 7.26 (d, $J=8.4 \mathrm{~Hz}, 4 \mathrm{H}, \mathrm{ArH}), 7.29$ (d, $J=$ $8.4 \mathrm{~Hz}, 4 \mathrm{H}, \mathrm{ArH}), 8.26$ (d, $J=4.8 \mathrm{~Hz}, 2 \mathrm{H}, \mathrm{PyH}) ;{ }^{13} \mathrm{C} \mathrm{NMR}$ $\left(125 \mathrm{MHz}, \mathrm{CDCl}_{3}\right) \delta: 51.59,77.14,111.37,127.81$, 128.63, 128.71, 132.49, 133.24, 135.22, 158.02, 162.90; IR (KBr) $v_{\text {max }}: 3351,2890,1615,1570,1501,1455,1379$, $972,821 \mathrm{~cm}^{-1}$; MS (EI) $m / z: 412[\mathrm{M}+\mathrm{H}]$.

$E$-2-(4'-氯苯基)-4-(5'-溴-2'-噻吩基)-1-(2'-嘧啶氨基) 丁烯-2-醇(2e): 收率 78\%, 白色晶体. m.p. 106 107 ${ }^{\circ} \mathrm{C}$ (from ethanol); ${ }^{1} \mathrm{H}$ NMR (500 MHz, $\left.\mathrm{CDCl}_{3}\right) \delta: 3.76$ (dd, $J=6.0,14.5 \mathrm{~Hz}, 1 \mathrm{H}, \mathrm{NCH}), 3.87$ (dd, $J=6.5,14.5 \mathrm{~Hz}, 1 \mathrm{H}$, $\mathrm{NCH}), 5.61(\mathrm{t}, J=6.0 \mathrm{~Hz}, 1 \mathrm{H}, \mathrm{NH}), 6.01(\mathrm{~s}, 1 \mathrm{H}, \mathrm{OH}), 6.25$ $(\mathrm{d}, J=15.5 \mathrm{~Hz}, 1 \mathrm{H}, \mathrm{CH}=\mathrm{CH}), 6.59(\mathrm{~d}, J=5.0 \mathrm{~Hz}, 1 \mathrm{H}$, PyH), 6.65 (d, $J=5.4 \mathrm{~Hz}, 1 \mathrm{H}, \mathrm{ThH}), 6.79$ (d, $J=15.5 \mathrm{~Hz}$, $1 \mathrm{H}, \mathrm{CH}=\mathrm{CH}), 6.87(\mathrm{~d}, J=5.4 \mathrm{~Hz}, 1 \mathrm{H}, \mathrm{ThH}), 7.32(\mathrm{~d}, J=$ $8.5 \mathrm{~Hz}, 2 \mathrm{H}, \mathrm{ArH}), 7.45$ (d, J=8.5 Hz, 2H, ArH), 8.24 (d, $J=5.0 \mathrm{~Hz}, 2 \mathrm{H}, \mathrm{PyH}) ;{ }^{13} \mathrm{C}$ NMR $\left(125 \mathrm{MHz}, \mathrm{CDCl}_{3}\right) \delta$ : $52.75,77.88,111.11,111.44,122.92,126.35,127.13$, $128.20,128.48,129.01,130.24,132.99,133.03,142.68$, 143.47, 157.94, 162.77; IR (KBr) $v_{\max }: 3428,1641,1589$, $1459,1401,1151,1094,618,491 \mathrm{~cm}^{-1}$. Anal. calcd for $\mathrm{C}_{18} \mathrm{H}_{15} \mathrm{BrClN}_{3} \mathrm{O}$ : C 49.50, H 3.46, N 9.62; found $\mathrm{C} 49.41$, H 3.47, N 9.4.

3.1.8 3E-1-(1',2',4'-三唑基)-2,4-二(4'-氯苯基)-1,3-丁 二烯 $(3 a)$ 的制备

用酮 $5 \mathbf{j}(7.2 \mathrm{mmol})$ 按第 3.1.6 节所述方法制备得到 的环氧化合物未经分离纯化, 立即溶解于 $50 \mathrm{~mL}$ 的 DMF 中, 再加入 $1,2,4-$ 三坐 $(8.6 \mathrm{mmol})$ 和氢氧化钠 $(8.6$ $\mathrm{mmol}), 50{ }^{\circ} \mathrm{C}$ 摚拌反应 $3 \mathrm{~h}$. 减压除去 DMF 后, 加入 50 $\mathrm{mL}$ 乙醚, 搅拌溶解, 并用饱和食盐水洗 3 次, 每次 30 $\mathrm{mL}$, 有机层干燥后, 蒸除乙醚得一无色稠状物. 加入 10 $\mathrm{mL}$ THF 溶解后, 加入 $1 \mathrm{~mL}$ 浓盐酸, 室温搅拌反应至白 色固体析出, 将其过滤, 乙醇重结晶得白色晶体 3a. 收 率 55\%, m.p. $143 \sim 144{ }^{\circ} \mathrm{C}$; ${ }^{1} \mathrm{H}$ NMR $(500 \mathrm{MHz}$, $\left.\left(\mathrm{CD}_{3}\right)_{2} \mathrm{SO}\right) \delta: 6.48(\mathrm{~d}, J=16.0 \mathrm{~Hz}, 1 \mathrm{H}, \mathrm{CH}=\mathrm{CH}), 7.31(\mathrm{~s}$, $1 \mathrm{H},=\mathrm{CHN}), 7.45(\mathrm{~d}, J=8.5 \mathrm{~Hz}, 2 \mathrm{H}, \mathrm{ArH}), 7.51(\mathrm{~d}, J=$ $8.4 \mathrm{~Hz}, 2 \mathrm{H}, \mathrm{ArH}), 7.53$ (d, $J=8.5 \mathrm{~Hz}, 2 \mathrm{H}, \operatorname{ArH}), 7.58$ (d, $J=8.4 \mathrm{~Hz}, 2 \mathrm{H}, \mathrm{ArH}), 8.21(\mathrm{~d}, J=16.0 \mathrm{~Hz}, 1 \mathrm{H}, \mathrm{CH}=\mathrm{CH})$, 8.37 (s, 1H,TrH), 8.94 (s, $1 \mathrm{H}, \operatorname{TrH}) ;{ }^{13} \mathrm{C}$ NMR [125 MHz, $\left.\left(\mathrm{CD}_{3}\right)_{2} \mathrm{SO}\right] \delta: 122.02,125.40,128.40,128.66,128.84$, $130.24,131.26,132.72,133.02,133.24,135.51,136.59$,
146.47, 152.56; IR (KBr) $v_{\max }: 3140,1641,1631,1492$, 1402, 1272, 1092, 781, $622 \mathrm{~cm}^{-1}$. Anal. calcd for $\mathrm{C}_{18} \mathrm{H}_{13} \mathrm{Cl}_{2} \mathrm{~N}_{3}$ : C 63.17, H 3.83, N 12.28; found C 63.22, H $3.83, \mathrm{~N} 12.25$.

3.1.9 3E-1-( $1^{\prime}, 2^{\prime}, 4^{\prime}$ - 三唑基)-2-(4'-氯苯基)-4-(5'-溴2'-噻吩基)-1,3-丁二烯(3b)的制备

用酮 5k $(7.2 \mathrm{mmol})$ 按第 3.1 .6 节所述方法制备得到 的环氧化合物未经分离纯化, 立即溶解于 $50 \mathrm{~mL}$ 的 DMF 中, 再加入 $1,2,4-$ 三唑 $(8.6 \mathrm{mmol})$ 和氢氧化钠 $(8.6$ $\mathrm{mmol}), 50{ }^{\circ} \mathrm{C}$ 搅拌反应 $3 \mathrm{~h}$. 减压除去 DMF 后, 加入 50 $\mathrm{mL}$ 乙醚, 搅拌溶解, 并用饱和食盐水洗 3 次, 每次 30 $\mathrm{mL}$, 有机层干燥后, 蒸除乙醚得一无色稠状物. 加入 10 $\mathrm{mL}$ THF 溶解后, 然后缓慢通入 $\mathrm{HCl}$ 气体, 室温反应, 反应大量放热, 析出白色固体, 将其过滤, 用乙醇重结 晶得白色晶体 3b, 收率 50\%. m.p. 146 147 ${ }^{\circ} \mathrm{C} ;{ }^{1} \mathrm{H}$ NMR [500 MHz, $\left.\left(\mathrm{CD}_{3}\right)_{2} \mathrm{SO}\right] \delta: 6.50(\mathrm{~d}, J=16.0 \mathrm{~Hz}, 1 \mathrm{H}$, $\mathrm{CH}=\mathrm{CH}), 6.97(\mathrm{~d}, J=3.8 \mathrm{~Hz}, 1 \mathrm{H}, \mathrm{ThH}), 7.14(\mathrm{~d}, J=3.8$ $\mathrm{Hz}, 1 \mathrm{H}, \mathrm{ThH}), 7.21(\mathrm{~s}, 1 \mathrm{H},=\mathrm{CHN}), 7.44(\mathrm{~d}, J=8.5 \mathrm{~Hz}$, 2H, ArH), 7.53 (d, $J=8.4 \mathrm{~Hz}, 2 \mathrm{H}, \mathrm{ArH}), 7.96$ (d, $J=16.0$ $\mathrm{Hz}, 1 \mathrm{H}, \mathrm{CH}=\mathrm{CH}), 8.12(\mathrm{~s}, 1 \mathrm{H}, \mathrm{TrH}), 8.30(\mathrm{~s}, 1 \mathrm{H}, \operatorname{TrH})$; ${ }^{13} \mathrm{C}$ NMR $\left[125 \mathrm{MHz},\left(\mathrm{CD}_{3}\right)_{2} \mathrm{SO}\right] \delta: 111.62,121.63,124.67$, 126.94, 128.67, 129.01, 131.26, 131.53, 133.01, 136.26, $143.85,146.48,152.59$; IR (KBr) $v_{\max }: 3118,1628,1602$, 1502, 1486, 1403, 1274, 1137, 1087, 673, $491 \mathrm{~cm}^{-1}$. Anal. calcd for $\mathrm{C}_{16} \mathrm{H}_{11} \mathrm{BrClN}_{3} \mathrm{~S}$ : C 48.94, H 2.82, N 10.70; found C 49.06, H 2.84, N 10.75 .

\section{2 抑菌活性测定}

\subsection{1 材料}

菌株: 结核分枝杆菌异烟肼单耐菌株 242, 为 WHO 质控菌株, 耐多药结核分枝杆菌 2312 和广泛耐药株 1220 , 来自上海市肺科医院临床分离培养. 液体培养基: Middlebrook 7H9 培养基干粉和营养添加剂(OADC)均 购自美国 BD 公司.

\subsection{2 实验方法}

受试菌株的制备 ${ }^{[37]}$ : 将受试菌株转入液体培养基, 于 $37{ }^{\circ} \mathrm{C}$ 培养 2 周, 吸取培养菌液少许, 置于 $4 \mathrm{~mL}$ 液体 培养基中, 加入直径 $2 \sim 3 \mathrm{~mm}$ 无菌玻璃珠 10 20粒, 振 荡 20 30 s, 静止沉淀 $10 \sim 20 \mathrm{~min}$, 吸取菌悬液上清, 用液体培养基调整比浊至 1 个麦氏单位, 相当于 1 $\mathrm{mg} / \mathrm{mL}$ 备用.

受试化合物的准备: 测试化合物用适量 DMSO 溶 解, $0.22 \mu$ 滤器过滤, 再以液体培养基稀释至所需实验 浓度. 受试药物均设置 12 个终浓度: $0.0625 、 0.125 、 0.25$ 、 $0.5 、 1 、 2 、 4 、 8 、 16 、 32 、 64$ 和 $128 \mu \mathrm{g} / \mathrm{mL}$.

操作步骤: 检测时, 各取上述化合物溶液 $100 \mu \mathrm{L}$, 
加到 96 孔微孔板中, 再加入 $10 \sim 3 \mathrm{mg} / \mathrm{mL}$ 浓度的菌液 $100 \mu \mathrm{L}$, 使化合物浓度达到设置的终浓度. 同一化合物 稀释度设三组平行对照. 另设溶剂梯度对照组, 不加化 合物对照组和不加化合物 $10 \%$ 接菌量对照组. $37{ }^{\circ} \mathrm{C}$ 培 养. 观察各化合物对结核分枝杆菌的最低抑菌浓度 $\left(\mathrm{MIC}_{90}\right)$. 实验结果如表 1 所示.

\section{辅助材料(Supporting Information) 目标化合物原始} 谱图. 这些材料可以免费从本刊网站(http://sioc-journal. $\mathrm{cn} /)$ 上下载.

\section{References}

[1] Boogaard, J.; Kibiki, G. S.; Kisanga, E. R.; Boeree, M. J.; Aarnoutse, R. E. Antimicrob. Agents Chemother. 2009, 53, 849.

[2] Palmer, B. D.; Thomposon, A. M.; Sutherland, H. S.; Blaser, A.; Kmentova, I.; Franzblau, S. G.; Wan, B. J.; Wang, Y. H.; Ma, Z. K.,; Denny, W. A. J. Med. Chem. 2010, 53, 282.

[3] Sethi, A.; Yadav, S.; Sharma, R. K. Int. J. Pharm. Sci. Rev. Res. 2010, 6, 117.

[4] Zhang, J.; Angala, S. K.; Pramanik, P. K.; Li. K.; Crick, D. C.; Liav, A.; Jozwiak, A.; Swiezewska, E.; Jackson, M.; Chatterjee, D. ACS Chem. Biol. 2011, 6, 819.

[5] Liu, Y. X.; Xiao, C. L.; Wang, Y. X.; Li, Y. H.; Yang, Y. H.; Li, Y. B.; Bi, C. W.; Gao, L. M.; Jiang, J. D.; Song, D. Q. Eur. J. Med. Chem. 2012, 52, 151.

[6] Oliveira, K. N.; Chiaradia, L. D.; Martins, P. G.; Mascarello, A.; Cordeiro, M. N.; Guido, R. V.; Andricopula, A. D.; Yunes, R. A.; Nunes, R. J.; Vernal, J.; Terenzi, H. Med. Chem. Commun. 2011, 2 , 500 .

[7] Srivastav, M. K.; Shantakumar, S. M. Chem. Sci. Trans. 2013, 2, 1056.

[8] Torres, E.; Moreno, E.; Ancizu, S.; Barea, C.; Galiano, S.; Aldana, I.; Monge, A.; Perez-Silanes, S. Bioorg. Med. Chem. Lett. 2011, 21, 3699 .

[9] Sasaki, H.; Haraguchi, Y.; Itotani, M.; Kuroda, H.; Hashizume, H.; Tomishige, T.; Kawasaki, M.; Masumoto, M.; Komatsu, M.; Tsubouchi, H. J. Med. Chem. 2006, 49, 7854.

[10] WHO Global Tuberculosis Report 2012. Available at: http://www.who.int/tb/publications/global_report/en/.

[11] Servusova, B.; Vobickova, J.; Paterova, P.; Kubicek, V.; kunes, J.; Dolezal, M.; Zitko, J. Bioorg. Med. Chem. Lett. 2013, 23, 3589.

[12] Ahsan, M. J.; Samy, J. G.; Khalilullah, H.; Bakht, M. A.; Hassan, M. Z. Eur. J. Med. Chem. 2011, 46, 5694.

[13] Manvar, A.; Bavishi, A.; Radadiya, A.; Patel, J.; Vora, V.; Dodia, N.; Rawal, K.; Shah, A. Bioorg. Med. Chem. Lett. 2011, 21, 4728.

[14] Simone, P. M.; Starke, J. R.; Vernon, A. A. Am. J. Respir. Crit. Care Med. 2003, 167, 62 .

[15] Saquib, M.; Gupta, M. K.; Sagar, R.; Prabhakar, Y. S.; Shaw, A. K.; Kumar, R.; Maulik, P. R.; Gaikwad, A. N.; Sinha, S.; Srivastava, A. K.; Chaturvedi, V.; Srivastava, R.; Srivastava, B. S. J. Med. Chem. 2007, 50, 2942.

[16] Huang, X.; Huang, H.; Li, H.; Sun, X.; Huang, H.; Lu, Y.; Long, Y.; She, Z. Org. Lett. 2013, 15, 721
[17] Koul, A.; Vranckx, L.; Dendouga, N.; Balmans, W.; Wyngaert, I.; Vergauwen, K.; Gohlmann, H.; Willebrords, R.; Poncelet, A.; Guillemont, J.; Bald, D.; Andries, K. J. Biol. Chem. 2008, 283, 25273.

[18] Dixit, P. P.; Patial, V. J.; Nait, P. S.; Jain, S.; Sinha, N.; Arora, S. K. Eur. J. Med. Chem. 2006, 41, 423.

[19] Pieter, C.; Davie, C.; Blaise, M. M.; Jan, J.; Birgit, M.; Vanessa, M.; Luc, V.; Kris, H.; Norbert, D. K. Eur. J. Med. Chem. 2013, 67, 98.

[20] Hwang, J.; Oh, T.; Kaneko, T.; Upton, A.; Franzblau, S.; Ma, Z.; Cho, S.; Kim, P. J. Nat. Prod. 2013, 76, 354.

[21] Ma, Z.; Lienhardt, C.; Mcllleron, H.; Nunn, A. J.; Wang, X. Lancet 2010, 275, 2100

[22] Chen, S.-J.; Cui, J.-G.; Li, Y.; Fan, L.-H. Chin. J. Org. Chem. 2011, 31,187 (in Chinese)

(陈思静，崔建国，李芗，范良华，有机化学, 2011, 31, 187.)

[23] Bo, Z.-S.; Wang, D.-X. The Research Development of New Heterocyclic Pesticides and Synthetic Method, Chemical Industry Press, Beijing, 2004, pp. 179 184 (in Chinese)

(柏再苏, 王大翔, 新杂环农药的研究发展及合成方法, 化学工 业出版社，北京, 2004, pp. 179 184.)

[24] Zhao, S. M.S. Thesis, East China Normal University, Shanghai, 2008 (in Chinese).

(赵升，硕士论文，华东师范大学，上海, 2008.)

[25] Zhang W.-S.; Li, A.-L. Medicinal Chemistry, Higher Education Press, Beijing, 1999, pp. 555 556 (in Chinese).

(伅文升, 李安良, 药物化学, 高等教育出版社, 北京, 1999, pp. $555 \sim 556$.)

[26] Merz, K. W.; Rauchle, K. Arch. Pharm. 1960, 16, 968.

[27] Okamoto, O.; Kobayashi, K.; Kawamoto, H.; Ito, S.; Satoh, A.; Kato, T.; Yamamoto, I.; Mizutani, S.; Hashimoto, M.; Shimizu, A.; Sakoh, H.; Nagatomi, Y.; Iwasawa, Y.; Takahashi, H.; Ishii, Y.; Ozaki, S.; Ohta, H. Bioorg. Med. Chem. Lett. 2008, 18, 3278.

[28] Baer, J. E.; Lockwood, R. G. J. Org. Chem. 1954, 76, 1162.

[29] Boyd, R. N.; Meadow, M. Anal. Chem. 1960, 32, 551.

[30] Luzzio, F. A. Synth. Commun. 1984, 14, 209.

[31] Finnegan, W. G.; Henry, R. A.; Lieber, E. J. Org. Chem. 1953, 75, 779.

[32] Herbst, R. M.; Roberts, C. W.; Harvill, E. J. J. Org. Chem. 1951, $16,139$.

[33] Rule, N. G.; Detty, M. R.; Kaeding, J. E.; Sinicropi, J. A. J. Org. Chem. 1995, 60, 1665 .

[34] Wei, X. C.; Du, Z. Y.; Zheng, X.; Cui, X. X.; Conney, A. H.; Zhang, K. Eur. J. Med. Chem. 2012, 53, 235.

[35] Corey, E. J.; Chaykovsky, M. J. Am. Chem. Soc. 1965, 87, 1353.

[36] Zakharychev, V. V.; Kuzenkov, A. V. Chem. Heterocycl. Compd. 2007, 43, 989.

[37] Wallace, R. J. Jr.; Nash, D. R.; Steele, L. C.; Steingrube, V. J. Clin. Microbiol. 1986, 24, 976

[38] Liang, G.; Zhou, H.; Wang, Y.; Gurley, E. C.; Feng, B.; Chen, L.; Xiao, J.; Yang, S.; Li, X. J. Cell. Mol. Med. 2009, 13, 3370.

[39] Weber, W. M.; Hunsaker, L. A.; Abcouwer, S. F.; Deck, L. M.; Vanderjagt, L. D. L. Bioorg. Med. Chem. 2005, 13, 3811.

[40] Sato, T.; Ino, H.; Fujitsuka, M.; Tanaka, K. Tetrahedron Lett. 1997, 38,6039 .

[41] Hazarkhani, H. Kumar, P.; Kondiram, K. S.; Shafi, G.; Ikhlas, M. S. G. Synthetic Communications 2010, 40, 2887.

[42] Musumarra, G.; Ballistreri, F. P. Org. Magn. Res. 1980, 14, 384. 